OPEN ACCESS

Edited by: Jana Seifert,

University of Hohenheim, Germany

Reviewed by:

Eva Pakostova, Coventry University, United Kingdom David Barrie Johnson, Bangor University, United Kingdom

*Correspondence:

Sabrina Hedrich sabrina.hedrich@bio.tu-freiberg.de

Specialty section: This article was submitted to Extreme Microbiology, a section of the journal

Frontiers in Microbiology

Received: 19 November 2021 Accepted: 17 December 2021

Published: 12 January 2022

Citation:

Malik L and Hedrich S (2022) Ferric Iron Reduction in Extreme Acidophiles.

Front. Microbiol. 12:818414. doi: 10.3389/fmicb.2021.818414

\section{Ferric Iron Reduction in Extreme Acidophiles}

\author{
Luise Malik and Sabrina Hedrich* \\ Research Group Biohydrometallurgy and Microbiology, Institute of Biosciences, TU Bergakademie Freiberg, Freiberg, \\ Germany
}

Biochemical processes are a key element of natural cycles occurring in the environment and enabling life on earth. With regard to microbially catalyzed iron transformation, research predominantly has focused on iron oxidation in acidophiles, whereas iron reduction played a minor role. Microbial conversion of ferric to ferrous iron has however become more relevant in recent years. While there are several reviews on neutrophilic iron reducers, this article summarizes the research on extreme acidophilic iron reducers. After the first reports of dissimilatory iron reduction by acidophilic, chemolithoautotrophic Acidithiobacillus strains and heterotrophic Acidiphilium species, many other prokaryotes were shown to reduce iron as part of their metabolism. Still, little is known about the exact mechanisms of iron reduction in extreme acidophiles. Initially, hypotheses and postulations for the occurring mechanisms relied on observations of growth behavior or predictions based on the genome. By comparing genomes of well-studied neutrophilic with acidophilic iron reducers (e.g., Ferroglobus placidus and Sulfolobus spp.), it became clear that the electron transport for iron reduction proceeds differently in acidophiles. Moreover, transcriptomic investigations indicated an enzymatically-mediated process in Acidithiobacillus ferrooxidans using respiratory chain components of the iron oxidation in reverse. Depending on the strain of At. ferrooxidans, further mechanisms were postulated, e.g., indirect iron reduction by hydrogen sulfide, which may form by disproportionation of elemental sulfur. Alternative scenarios include Hip, a high potential iron-sulfur protein, and further cytochromes. Apart from the anaerobic iron reduction mechanisms, sulfur-oxidizing acidithiobacilli have been shown to mediate iron reduction at low $\mathrm{pH}(<1.3)$ under aerobic conditions. This presumably non-enzymatic process may be attributed to intermediates formed during sulfur/tetrathionate and/or hydrogen oxidation and has already been successfully applied for the reductive bioleaching of laterites. The aim of this review is to provide an up-to-date overview on ferric iron reduction by acidophiles. The importance of this process in anaerobic habitats will be demonstrated as well as its potential for application.

Keywords: extreme acidophiles, iron reduction, biochemistry, biohydrometallurgy, reductive bioleaching 


\section{LIFESTYLE OF ACIDOPHILIC MICROORGANISMS}

Acidic environments such as acid mine drainage are the result of anthropogenic influences or have a natural origin, e.g., solfataric springs (Johnson, 2009). Depending on the occurring $\mathrm{pH}$, these environments are habitats for moderate $(\mathrm{pH} 3-5)$ or extreme acidophilic $(\mathrm{pH}<3)$ organisms. Representatives of all three domains have been identified under acidic conditions, also eukaryotes, mainly fungi and algae, some rotifers, ciliates and species of the genus Euglena, were determined in acidic waterbodies (Quatrini and Johnson, 2016; Stolz, 2017; Johnson and Quatrini, 2020). However, this article will focus on acidophilic prokaryotes, which are well adapted to the challenging conditions of extremely low $\mathrm{pH}$ habitats (Dopson, 2016; Golyshina et al., 2016).

\section{Adaptation to Extreme Environments}

Most of the challenges acidophiles have to face with respect to their metabolism and morphological adaptations, are dictated by the conditions in their extreme environment. Low $\mathrm{pH}$ values are characterized by high concentrations of hydronium ions caused by a combination of proton-producing biotic and abiotic reactions, for instance, the generation of sulfuric acid in mineimpacted and geothermal areas (Johnson, 2009). Acidophiles have evolved appropriate mechanisms to overcome this cell homeostasis-threatening issue, e.g., the presence of a reverse positive inner membrane potential (Matin, 1999). With respect to highly acidic habitats, high temperatures are also an important influence, as these two quite hostile conditions occasionally appear together, for example, in hot solfatara springs or volcanoes (Johnson, 2009). Besides high concentrations of protons, a variety of metal cations is present in most acidic environments. These are generated by mineral leaching processes, which cause mobilization of partially toxic metal and metalloid cations (Gadd, 2009). The cations present, especially iron and copper, can in turn generate reactive oxygen species, e.g., through the Fenton reaction, which increases the oxidative stress and causes damage of cellular components (Ferrer et al., 2016). However, the inorganic compounds released during leaching processes are also a source of energy for many microorganisms. This is of particular importance since most acidic environments have low levels of organic carbon sources which forced the development of widespread abilities to utilize autotrophic metabolisms (Johnson, 2009).

\section{Metabolic Diversity of Extreme Acidophiles}

Despite the fact that acidophiles are to some extent constrained by their environmental conditions, diverse metabolisms have evolved that are used by these organisms (Figure 1; Johnson, 1998). Acidophiles can exhibit both obligate heterotrophic or obligate/facultative autotrophic carbon metabolisms, with the latter using oxidation or reduction of elemental sulfur, iron or hydrogen to generate ATP (Johnson and Hallberg, 2008; Johnson, 2009). Some acidophiles use a wide range of dissimilation pathways, while the obligate aerobic autotrophic Leptospirillum is only capable of oxidizing ferrous iron (Dopson, 2016). Depending on the utilized electron acceptor and donor, the acidophilic electron transport systems differ considerably. Especially in the case of sulfur metabolism, the variety of occurring oxidation states ( -2 to +6 ) allow several pathways by which reduced inorganic sulfur compounds (RISC) are partially or completely oxidized to sulfate. Nevertheless, a lot of effort was put in the elucidation of the underlying enzymatic processes, as reviewed elsewhere (Wang et al., 2019; Dahl, 2020). In addition to the well-studied sulfur metabolism, hydrogen oxidation under anaerobic or aerobic conditions is also a common feature of acidophiles (Hedrich and Johnson, 2013b; Kucera et al., 2020).

Regarding the iron oxidation mechanism in acidophiles, different strategies evolved simultaneously in several phyla, e.g., $\gamma$-Proteobacteria, Firmicutes, Nitrospira, Crenarchaeota, and Euryarchaeota (Ilbert and Bonnefoy, 2013). In particular, for Acidithiobacillus ferrooxidans, bioinformatic, genetic, proteomic, and transcriptomic investigations of the electron transfer chain and related genes led to a widely accepted iron oxidation model (Ingledew, 1982; Bonnefoy, 2010; Amouric et al., 2011; Kucera et al., 2013; Jiang et al., 2021). This is still work in progress, as the results obtained on an ongoing basis extend the previously postulated iron oxidation mechanism to some extent, for example through newly discovered gene clusters (Ai et al., 2018). Nevertheless, the acknowledged electron transport chain from iron to the terminal electron acceptor includes an outer membrane cytochrome $c$ (Cyc2), transferring the electron from iron to rusticyanin, a copper protein situated in the periplasm (Giudici-Orticoni et al., 1999; Yarzábal et al., 2002). Depending on its destination, the electron is further transported to the NADH-1 complex via cytochrome $c$ (CycA1), the cytochrome $b c_{1}$ complex and ubiquinone pool to produce reduction equivalents (Elbehti et al., 1999, 2000). Electron transport along the thermodynamic gradient to reduce oxygen to water occurs, if the electron is transferred from rusticyanin to cytochrome $c$ (Cyc1) and further to the $a a_{3}$ cytochrome oxidase (Kai et al., 1992; Malarte et al., 2005). Accordingly, the components of the iron oxidation chain in Acidithiobacillus ferrooxidans form a supercomplex extending from the outer membrane integrated Cyc2 through the periplasm to the cytochrome oxidase, $b c_{1}$ complex, and NADH-1 complex in the inner membrane (Figure 2; Ilbert and Bonnefoy, 2013).

Many acidophiles are facultative anaerobes, preferentially using oxygen as electron acceptor, but in its absence, they may alternatively reduce iron or rarely sulfur (Gyure et al., 1990; Coupland and Johnson, 2008). Dissimilatory ferric iron reduction under anaerobic conditions is widespread among acidophiles and can be coupled to the oxidation of hydrogen, sulfur, RISC or organic compounds (Coupland and Johnson, 2008; Johnson, 2010). The greater iron solubility at low $\mathrm{pH}$ and its abundance in many acidic environments are reasons why iron is used by acidophiles as both electron acceptor and donor (Johnson, 2009). Additionally, the much higher redox potential of the ferrous/ferric iron pair at low $\mathrm{pH}(+663 \mathrm{mV}, \mathrm{pH} 2$, sulfaterich solution) (Johnson et al., 2017) makes it a more feasible electron acceptor compared to neutral conditions $(+200 \mathrm{mV}$, 


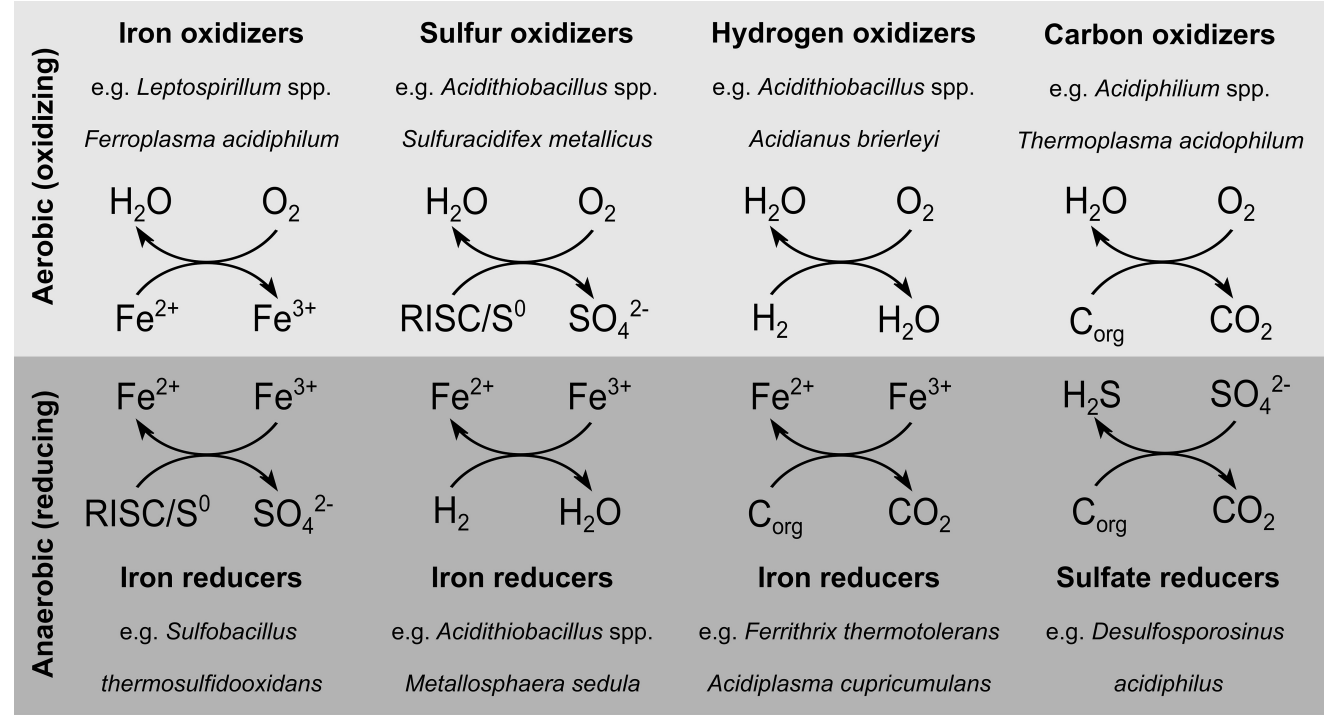

FIGURE 1 | Diversity of dissimilation pathways used by acidophiles. The underlying data can be found in the associated studies (Hedrich et al., 2011 ; Hedrich and Johnson, 2013b; Dopson, 2016). RISC, reduced inorganic sulfur compounds; Corg, organic carbon.

$\mathrm{pH} \mathrm{7,} \mathrm{bicarbonate-containing} \mathrm{environments)} \mathrm{(Ehrenreich} \mathrm{and}$ Widdel, 1994). Accordingly, microbial cycling of iron can occur between habitat niches of different dissolved oxygen levels, which contributes significantly to the natural iron cycle.

\section{IRON REDUCTION BY ACIDOPHILES}

Iron reduction as part of the natural iron cycle plays an important role on Earth (Kappler and Straub, 2005). Despite the well-studied mechanisms of iron reduction by neutrophilic microorganisms, the process in acidophilic microbes has not yet been sufficiently explored. Nevertheless, genome comparisons of known neutrophilic iron reducers with those of acidophiles revealed few similarities suggesting a distinct mechanism in acidophiles (Masaki et al., 2018). Depending on the considered microorganism, ferric iron reduction by acidophiles can take place under anaerobic, aerobic as well as micro-aerobic conditions. The most important milestones of ferric iron reduction research in acidophiles are summarized in Table 1. First investigations of acidithiobacilli described aerobic ferric iron reduction at low pH (Brock and Gustafson, 1976; Sand, 1989) and anaerobic ferrous iron formation (Brock and Gustafson, 1976). Micro-aerobic iron reduction was especially studied for the genus Acidiphilium (Johnson and Bridge, 2002). Regarding archaea, only a few selected genera have been studied profoundly with respect to their iron reduction mechanism, most notably the anaerobic and micro-aerobic studies of Sulfolobus, Sulfuracidifex, and Saccharolobus (latter no longer termed as extreme acidophile due to a $\mathrm{pH}$ optimum of 4.5) (Masaki et al., 2018). Moreover, investigations of the archeon "Ferroplasma acidarmanus" Fer1 revealed anaerobic ferric iron reduction rates which were comparable with those determined for Acidiphilium species (Dopson et al., 2007). Overall, ferric iron reduction was shown for different physiologic groups of acidophilic microorganisms, covering heterotrophs and chemoautotrophs which feature temperature optima ranging from mesophilic to thermophilic adaptations (Johnson and Bridge, 2002). In particular, the ability of acidophiles to mediate reductive dissolution of ferric iron minerals is frequently explored, with emphasis on the applicability prior to elucidation of the exact biochemical mechanism (Hallberg et al., 2011a; Nancucheo et al., 2014; Norris et al., 2015; Marrero et al., 2017). However, certain research groups provided insight into the mechanisms of ferric iron reduction that occur in extreme acidophiles at different oxygen concentrations, which will be discussed in more detail.

\section{Anaerobic Iron Reduction at Low pH}

After the observation of iron-reducing processes by numerous neutrophilic prokaryotes (Bromfield, 1954; Ottow and Glathe, 1971; Lovley, 1991), anaerobic iron reduction by extreme acidophiles was first described in 1976, for the species Acidithiobacillus ferrooxidans (Brock and Gustafson, 1976). Under the given conditions, elemental sulfur-respiring At. ferrooxidans reduced iron exclusively in the absence of oxygen. Accordingly, it was hypothesized that ferric iron may serve as an alternative electron acceptor for acidophiles in anaerobic habitats. To date, iron reduction in the absence of oxygen has been demonstrated for a variety of moderate and extremely acidophilic microorganisms. The phylogenetically heterogenous group of iron-reducing acidophiles includes archaeal as well as bacterial species covering the phyla Crenarchaeota, Euryarchaeota, Acidobacteria, Actinobacteria, Firmicutes, and Proteobacteria (Tables 2-5).

The investigation of the ferric iron reduction mechanisms in acidophiles is still ongoing and only few organisms, e.g., Acidithiobacillus ferrooxidans, were examined in detail. Over the 


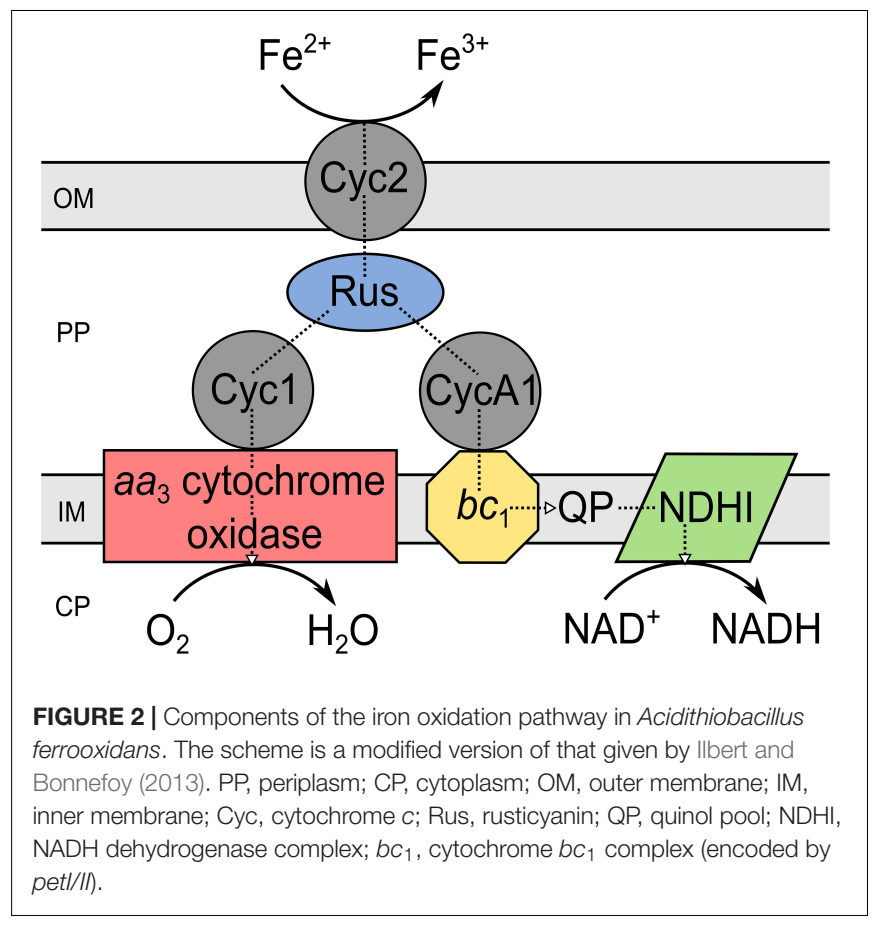

years, various hypothesis were given, for instance an involvement of a nitrate reductase or tetrathionate hydrolase (Johnson and McGinness, 1991; Sugio et al., 2009), which could not be confirmed by later studies (Kucera et al., 2012, 2016b; Osorio et al., 2013). In addition to the biochemical process, it was crucial to determine whether anaerobic iron reduction is accompanied by cell growth or merely a mechanism for maintaining cellular functions. Initially, no growth was detectable for anaerobic ironreducing At. ferrooxidans (Sugio et al., 1988), which was later contributed to an insufficient carbon dioxide supply (Das et al., 1992). Soon, anaerobic dissimilatory iron reduction was reported for different At. ferrooxidans strains (Pronk et al., 1991, 1992; Das et al., 1992). In this context, a doubling time of $24 \mathrm{~h}$ was determined for ferric iron-respiring At. ferrooxidans ${ }^{T}$ on elemental sulfur (Pronk et al., 1992) and the existence of two distinct electron transport systems for ferric iron reduction was suggested (Das et al., 1992). Additionally, the electron donor provided during pre-cultivation seemed to have a major impact on the ferric iron reduction ability of At. ferrooxidans. Cells grown on elemental sulfur for several generations were not able to reduce ferric iron when changed to anaerobic cultivation conditions, while the ones pre-cultivated with ferrous iron were (Kucera et al., 2012).

\section{Uncovering Anaerobic Ferric Iron Reduction Mechanisms in Acidithiobacilli}

All to date described extreme acidophilic iron-oxidizing acidithiobacilli have been identified as anaerobic ferric iron reducers (Pronk et al., 1992; Hallberg et al., 2010; Hedrich and Johnson, 2013a; Falagán and Johnson, 2016; Norris et al., 2020). Accordingly, this genus offers great potential for research into the biochemical mechanisms of iron reduction, especially because Acidithiobacillus ferrooxidans has been studied in detail for its molecular processes as a flagship organism of bioleaching. Since the first investigation of anaerobic ferric iron reduction by At. ferrooxidans in the 1970s, a lot of effort has been put into the elucidation of the underlying enzymatic process. Sugio et al. $(1985,1987)$ purified and to some extent characterized a sulfur:ferric iron oxidoreductase from ferrous iron-grown At. ferrooxidans AP19-3. This periplasmic space enzyme catalyzed the oxidation of elemental sulfur to sulfite while reducing ferric to ferrous iron as one step of the sulfur metabolism and was therefore seen as an important part of the iron reduction chain (Sugio et al., 1985, 1987). In the same year, Corbett and Ingledew presented a model for the anaerobic iron respiration pathway of At. ferrooxidans ${ }^{\mathrm{T}}$ (Figure 3) utilizing complexes and electron transporters of the aerobic iron oxidation chain (section "Metabolic Diversity of Extreme Acidophiles"). Thereby, the electron transfer from the donor elemental sulfur to the terminal acceptor iron may involve the cytochrome $b c_{1}$ complex, cytochrome $c$, rusticyanin, cytochrome oxidase, cytochrome $a_{1}$, and other transporters of the ferrous iron oxidation pathway located in the periplasmic space (Corbett and Ingledew, 1987). With this, the hypothesis arose that components of the iron oxidation chain could also be used in reverse for ferric iron respiration in acidophiles under anaerobic conditions, which was further investigated since then. Additional evidence was given by the observation, that At. ferrooxidans lacked the ability of anaerobic iron reduction after six consecutive aerobic cultivations on sulfur, which was contributed to the absence of some iron oxidation chain components in sulfur adapted cells (Kucera et al., 2012). Further, the investigation of anaerobic ferric iron respiration by At. ferrooxidans JCM 7811, later renamed as At. ferriphilus JCM 7811 (Kucera et al., 2020), with varying electron donors (hydrogen or elemental sulfur) resulted in the production of high amounts of a new c-type cytochrome (Ohmura et al., 2002). The purified cytochrome differed from other At. ferrooxidans-originating ones by an intermediate midpoint potential of about $+560 \mathrm{mV}(\mathrm{pH} 2.0)$ and was shown to act as an electron acceptor for ferric iron reduction (Ohmura et al., 2002). Consequently, the evidence was growing that cytochromes may play a major role in electron transfer during ferric iron reduction by sulfur-respiring acidophiles. The increased abundance of rusticyanin and cytochrome Cyc1 (both part of the ferrous iron oxidation pathway and encoded by the rus operon) in ferric iron-respiring At. ferrooxidans CCM 4253 further confirmed the contribution of rus-encoded, iron oxidation-related proteins in the iron reduction process (Kucera et al., 2012).

In 2013, Osorio et al. (2013) suggested two models for the anaerobic ferric iron reduction in At. ferrooxidans ${ }^{\mathrm{T}}$ (Figure 3), based on microarray and proteomic examination during aerobic and anaerobic growth. One supported the aforementioned reverse utilization of the iron oxidation chain, by proposing the involvement of the $c_{4}$-type cytochrome CycA2 and the $b c_{1}$ complex. The second model introduced a new aspect, stating that an enzymatic disproportionation of sulfur during anaerobic sulfur oxidation may produce $\mathrm{H}_{2} \mathrm{~S}$ which could mediate ferric iron reduction at low pH (Osorio et al., 2013). 
TABLE 1 | Most important discoveries during research of iron reduction in acidophiles.

\begin{tabular}{|c|c|c|}
\hline Year & Investigation & References \\
\hline 1976 & $\begin{array}{l}\text { First description of ferric iron reduction by acidophilic bacteria and archaea: } \\
\text { - Acidithiobacillus thiooxidans (aerobic) } \\
\text { - Acidithiobacillus ferrooxidans (anaerobic) } \\
\text { - Sulfolobus acidocaldarius (aerobic) }\end{array}$ & Brock and Gustafson, 1976 \\
\hline 1987 & Purification and characterization of a sulfur:ferric iron oxidoreductase from ferrous iron-grown At. ferrooxidans & Sugio et al., 1987 \\
\hline 1987 & $\begin{array}{l}\text { First model of anaerobic iron respiration pathway of } A t \text {. ferrooxidans utilizing transporters of the ferrous iron } \\
\text { oxidation chain (cytochrome } b c_{1} \text { complex, cytochrome } c \text {, rusticyanin, cytochrome oxidase, cytochrome } a_{1} \text { ) }\end{array}$ & Corbett and Ingledew, 1987 \\
\hline 1989 & Aerobic iron reduction by resting cells of $A t$. ferrooxidans at extremely low pH & Sand, 1989 \\
\hline 1991 & Anaerobic dissimilatory iron reduction by At. ferrooxidans & Pronk et al., 1991 \\
\hline 1991 & Aerobic dissimilatory iron reduction by heterotrophic Acidiphilium spp. & Johnson and McGinness, 1991 \\
\hline 1998 & $\begin{array}{l}\text { Iron reduction by moderately thermophilic iron-oxidizing bacteria: } \\
\text { - Sulfobacillus thermosulfidooxidans } \\
\text { - Sulfobacillus acidophilus } \\
\text { - Acidimicrobium ferrooxidans } \\
\text { Iron cycling in static, aerobic cultures }\end{array}$ & Bridge and Johnson, 1998 \\
\hline 2002 & Distinction between a constitutive and oxygen-inducible iron reduction system in Acidiphilium spp. & Johnson and Bridge, 2002 \\
\hline 2011 & First demonstration of using iron reduction by acidophiles for mineral processing and metal extraction & Hallberg et al., 2011a \\
\hline 2013 & Suggestion of a second, indirect iron reduction mechanism in At. ferrooxidans mediated by $\mathrm{H}_{2} \mathrm{~S}$ at low pH & Osorio et al., 2013 \\
\hline 2015 & $\begin{array}{l}\text { Indication of an indirect, non-growth-related iron reduction mechanism in At. thiooxidans at aerobic, extreme } \\
\text { acidic conditions }\end{array}$ & Marrero et al., 2015 \\
\hline 2016 & Hypothetic iron reduction pathway in At. ferrooxidans including a high potential iron-sulfur protein & Kucera et al., $2016 a$ \\
\hline 2020 & Model for anaerobic iron reduction coupled to hydrogen oxidation in At. ferrooxidans & Kucera et al., 2020 \\
\hline 2021 & Introduction of the term "latent iron reduction" for acidithiobacilli & Johnson et al., 2021 \\
\hline
\end{tabular}

However, the ability of acidithiobacilli to grow anaerobically by coupling hydrogen oxidation (no $\mathrm{H}_{2} \mathrm{~S}$ production) to iron reduction (Hedrich and Johnson, 2013b) suggested that ferric iron reduction mechanisms other than the indirect one must exist (Osorio et al., 2013). Still, the existence of an indirect iron reduction mechanism by $\mathrm{H}_{2} \mathrm{~S}$ was not confirmed for At. ferrooxidans CCM 4253 due to no detection of $\mathrm{H}_{2} \mathrm{~S}$ with lead acetate and a repressed expression of the sulfur reductaseencoding sre operon during anaerobic growth (Kucera et al., 2016a). Besides, multiple ferric iron reduction mechanisms were again indicated during comparative examination of strain CCM 4253 with and without ferric iron reduction ability (Kucera et al., 2015). Proteomic results showed 150 repressed spots of important proteins involved in the iron oxidation (e.g., rusticyanin, cytochrome Cyc2) and sulfur metabolism for cells lacking the iron reduction ability (Kucera et al., 2015). The induction of rusticyanin and $\mathrm{Cyc} 2$ in anaerobic ironsulfur-respiring At. ferrooxidans implied that electrons may be collected by rusticyanin and transported to the outermembrane cytochrome which could act as terminal ferric iron reductase (Kucera et al., 2015, 2016b). Moreover, transcriptional investigation of strain CCM 4253 coupling iron reduction to anaerobic sulfur oxidation revealed an overexpression of the hip gene encoding a high potential iron-sulfur protein (Hip) and thereby a new hypothetic iron reduction pathway in At. ferrooxidans (Kucera et al., 2016a). During aerobic growth, this iron-sulfur protein enables electron transfer between the quinone pool and the final oxidase, which reduces oxygen (Quatrini et al., 2006). Accordingly, the predicted, Hip-including anaerobic iron reduction mechanism suggested a transfer of electrons obtained through sulfur oxidation to take place from the quinone pool to the $b c_{1}$ complex and is further transported by $\mathrm{CycA} 2$ and Hip to an unknown iron reductase (Kucera et al., 2016a; Figure 3). It has to be taken in consideration, that the pre-cultures used during the aforementioned anaerobic ferric iron reduction examinations were aerobically ferrous irongrown, which invited Norris et al. (2018) to perform proteomic studies with anaerobically adapted cultures. Thereby, two further cytochromes, Сyc2B and $\mathrm{Cyc1B}$, were detected and the allover results supported a participation of petII-encoded proteins $\left(b c_{1}\right.$ complex II and CycA2) in the anaerobic ferric iron reduction of sulfur-oxidizing At. ferrooxidans ${ }^{\mathrm{T}}$ (Norris et al., 2018). This in turn reinforces the alternative ferric iron reduction mechanism using a high potential iron-sulfur protein as predicted by Kucera et al. (2016a).

To date, all the given insights into the biochemical iron reduction mechanism coupled to sulfur oxidation indicate the occurrence of at least two enzymatic, species-dependent iron reduction mechanisms in acidithiobacilli (Figure 3). The existence of an indirect process mediated by $\mathrm{H}_{2} \mathrm{~S}$ needs further investigation, as the contradicting results of different strains do not imply a tendency. Nevertheless, there is a lot of evidence for both suggested electron transport chains from the inner to the outer membrane which may transfer electrons from the quinone pool to (i) the final reductase Cyc2 via $b c_{1}$ complex I, CycA1, and rusticyanin; or (ii) a still unknown reductase by $b c_{1}$ complex II, CycA2, and Hip (Corbett and Ingledew, 1987; Ohmura et al., 2002; Kucera et al., 2012, 2015, 2016a,b; Osorio et al., 2013; Norris et al., 2018). Analogous to ferric iron reduction during sulfur oxidation, the proposed mechanisms 
TABLE 2 | Ferric iron-reducing extreme acidophilic archaea.

\begin{tabular}{|c|c|c|c|c|c|c|c|}
\hline Organism & $\begin{array}{l}\mathrm{Fe} \\
\text { ox. }\end{array}$ & $\begin{array}{l}\mathrm{S}^{0} \\
\mathrm{ox}\end{array}$ & $\mathrm{pH}$ & $\begin{array}{l}\text { Temp. } \\
{\left[{ }^{\circ} \mathrm{C}\right]}\end{array}$ & Conditions Fe red., ED & Growth & References \\
\hline Acidianus brierleyi ${ }^{T}$ & + & + & $\begin{array}{l}1.5-2.0 \\
1.0-6.0\end{array}$ & $\begin{array}{c}70 \\
45-75\end{array}$ & Anaerobic: $\mathrm{H}_{2} \mathrm{~S}$ (w/, w/o OC) & + & Plumb et al., 2007 \\
\hline "Acidianus copahuensis" & + & + & $\begin{array}{l}2.5-3.0 \\
1.0-5.0\end{array}$ & $\begin{array}{c}75 \\
55-80\end{array}$ & Anaerobic: $\mathrm{S}^{0}$ or $\mathrm{H}_{2}$ & + & Giaveno et al., 2013 \\
\hline "Acidianus manzaensis"T & - & + & $\begin{array}{l}1.2-1.5 \\
1.0-5.0\end{array}$ & $\begin{array}{c}80 \\
60-90\end{array}$ & Anaerobic: $\mathrm{S}^{0}, \mathrm{H}_{2}$ or $\mathrm{OC}$ & + & Yoshida et al., 2006 \\
\hline Acidianus sulfidivorans ${ }^{\top}$ & + & + & $\begin{array}{c}0.8-1.4 \\
0.35-3.0\end{array}$ & $\begin{array}{c}74 \\
45-83\end{array}$ & Anaerobic: $\mathrm{H}_{2} \mathrm{~S}$ (w/, w/o OC) & + & Plumb et al., 2007 \\
\hline Acidiplasma aeolicum ${ }^{\top}$ & + & RISC & $\begin{array}{c}1.4-1.6 \\
0-4.0\end{array}$ & $\begin{array}{l}42-45 \\
15-65\end{array}$ & Anaerobic: OC & + & Golyshina et al., 2009 \\
\hline Acidiplasma cupricumulans $^{\top}$ & + & - & $\begin{array}{l}1.0-1.2 \\
>0.4\end{array}$ & $\begin{array}{c}53.6 \\
22-63\end{array}$ & Anaerobic: $\mathrm{K}_{2} \mathrm{~S}_{4} \mathrm{O}_{6}$ w/ OC & + & Hawkes et al., 2006 \\
\hline "Ferroplasma acidarmanus" & + & ND & $\begin{array}{c}1.2 \\
0.2-2.5\end{array}$ & $\begin{array}{c}42 \\
23-46\end{array}$ & Anaerobic: OC & + & Dopson et al., 2004 \\
\hline $\begin{array}{l}\text { Ferroplasma acidiphilum }{ }^{\top} \text {, } \\
\text { DR1 and MT17 }\end{array}$ & + & $-\mathrm{RISC}$ & $\begin{array}{c}1.7 \\
1.3-2.2\end{array}$ & $\begin{array}{c}35 \\
20-45\end{array}$ & Anaerobic: OC & + & $\begin{array}{l}\text { Dopson et al., 2004; } \\
\text { Johnson, } 2010\end{array}$ \\
\hline "Ferroplasma thermophilum" & + & - & $\begin{array}{c}1.0 \\
0.2-2.5\end{array}$ & $\begin{array}{c}45 \\
30-60\end{array}$ & Anaerobic: OC & + & Zhou et al., 2008 \\
\hline Metallosphaera sedula ${ }^{\top}$ & + & + & $1.0-4.5$ & $\begin{array}{c}75 \\
50-80\end{array}$ & Aerobic: $\mathrm{H}_{2}$ & ND & Auernik and Kelly, 2010 \\
\hline Saccharolobus caldissimus $^{\top}$ & $\mathrm{FeS}_{2}$ & - & $\begin{array}{c}3.0 \\
1.5-6.0\end{array}$ & $\begin{array}{c}85 \\
65-93\end{array}$ & Anaerobic: OC & + & Sakai and Kurosawa, 2018 \\
\hline Saccharolobus shibatae ${ }^{\top}$ & $\mathrm{FeS}_{2}$ & - & $\begin{array}{c}3.0 \\
1.5-6.0\end{array}$ & $\begin{array}{c}81 \\
55-86\end{array}$ & Anaerobic: OC & + & Sakai and Kurosawa, 2018 \\
\hline Sulfolobus acidocaldarius ${ }^{\top}$ & + & + & $\begin{array}{l}2.0-3.0 \\
1.0-5.9\end{array}$ & $\begin{array}{l}75-80 \\
55-80\end{array}$ & $\begin{array}{c}\text { Aerobic: } \mathrm{S}^{0} \text { or } \mathrm{OC} \\
\text { Microaerobic: } \mathrm{OC} \\
\text { Anaerobic: } \mathrm{OC}\end{array}$ & $\begin{array}{c}- \\
N D \\
-\end{array}$ & $\begin{array}{l}\text { Brock and Gustafson, } \\
\text { 1976; Masaki et al., } 2018\end{array}$ \\
\hline Sulfuracidifex metallicus ${ }^{\top}$ & + & + & $\begin{array}{l}2.0-3.0 \\
1.0-4.5\end{array}$ & $\begin{array}{c}65 \\
50-75\end{array}$ & Anaerobic: $\mathrm{S}^{0}$ & - & Masaki et al., 2018 \\
\hline Sulfurisphaera ohwakuensis ${ }^{\top}$ & $\mathrm{FeS}_{2}$ & + & $\begin{array}{c}2.0 \\
1.5-6.0\end{array}$ & $\begin{array}{c}84 \\
60-91\end{array}$ & Anaerobic: OC & + & Tsuboi et al., 2018 \\
\hline Sulfurisphaera tokodaii ${ }^{\mathrm{T}}$ & + & + & $\begin{array}{l}2.5-3.0 \\
1.5-6.0\end{array}$ & $\begin{array}{c}80 \\
60-96\end{array}$ & $\begin{array}{c}\text { Anaerobic: } \mathrm{S}^{0} \text { or } \mathrm{OC} \\
\text { Micro-aerobic: OC }\end{array}$ & $\begin{array}{c}+ \\
N D\end{array}$ & Masaki et al., 2018 \\
\hline Thermoplasma acidophilum ${ }^{\top}$ & ND & - & $\begin{array}{c}1.0-2.0 \\
0.5-34.0\end{array}$ & $\begin{array}{c}59 \\
45-63\end{array}$ & Anaerobic: $\mathrm{S}^{0}$ & ND & Zhang et al., 2018 \\
\hline
\end{tabular}

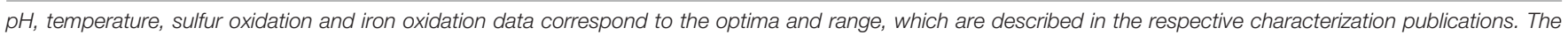

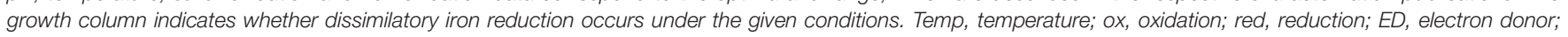
ND, no data; OC, organic carbon.

of anaerobic iron reduction coupled to hydrogen oxidation are only partially understood and are largely based on proteomic and transcriptomic analyses. In 2020, Kucera et al. (2020) introduced a model for anaerobic hydrogen metabolism in At. ferrooxidans CCM 4253 which suggests an electron transfer from hydrogen to iron via [NiFe] hydrogenase, ubiquinone pool, $b c_{1}$ complex, cytochrome $c_{4}$, Cycl or Hip, rusticyanin, and Cyc2. Consequently, the mechanisms that enable ferric iron reduction in an anaerobic environment may involve several similar enzymes and proteins which are also part of the ferrous iron oxidation pathway in acidophiles, regardless of the electron donor used (Figure 4). Therefore, it is likely, that organisms lacking these important components in their electron transport chain of iron oxidation (e.g., rus-encoded proteins), may not be able to enzymatically reduce ferric iron. However, there is still great research potential concerning these iron reduction processes. Only well-understood biochemical mechanisms can be optimally adapted to applications and supported by the required environmental conditions. Moreover, a detailed insight into the underlying microbial processes is key for the assessment of their large-scale implementation as biohydrometallurgical processes for reducing ferric iron minerals, such as goethite.

\section{Iron Reduction Under Micro-Aerobic Conditions}

Besides iron-reducing processes under oxic or anoxic conditions, some acidophilic, heterotrophic microorganisms reduce ferric iron and/or mediate reductive dissolution of ferric iron minerals under micro-aerobic conditions. The type strains of Acidibacter ferrireducens, Acidicapsa acidiphila, and Acidicapsa ferrireducens catalyze the reductive dissolution of schwertmannite while metabolizing organic carbon sources (glucose, yeast extract) under oxygen-limitation (Falagán and Johnson, 2014; 
TABLE 3 | Ferric iron-reducing extreme acidophilic autotrophic bacteria.

\begin{tabular}{|c|c|c|c|c|c|c|c|}
\hline Organism & $\begin{array}{l}\text { Fe } \\
\text { ox. }\end{array}$ & $\begin{array}{l}S^{0} \\
o x\end{array}$ & $\mathrm{pH}$ & $\begin{array}{l}\text { Temp. } \\
{\left[{ }^{\circ} \mathrm{C}\right]}\end{array}$ & Conditions Fe red., ED & Growth & References \\
\hline Acidiferrobacter thiooxydans ${ }^{\top}$ & + & + & $\begin{array}{c}2.0 \\
>1.2\end{array}$ & $\begin{array}{c}38 \\
<47\end{array}$ & Anaerobic: $\mathrm{S}^{0}$ & + & Hallberg et al., 2011b \\
\hline Acidithiobacillus caldus ${ }^{\top}$ & - & + & $\begin{array}{l}2.0-2.5 \\
1.0-3.5\end{array}$ & $\begin{array}{c}45 \\
32-52\end{array}$ & Aerobic: $\mathrm{S}^{0}$ & - & Johnson et al., 2017 \\
\hline Acidithiobacillus ferrianus ${ }^{\top}$ & + & + & 2.0 & 30 & Anaerobic: $\mathrm{S}^{0}$ or $\mathrm{H}_{2}$ & + & Norris et al., 2020 \\
\hline Acidithiobacillus ferridurans $^{\top}$ & + & + & $\begin{array}{c}2.1 \\
<1.3\end{array}$ & 29 & $\begin{array}{c}\text { Anaerobic: } \mathrm{S}^{0}, \mathrm{~K}_{2} \mathrm{~S}_{4} \mathrm{O}_{6} \text { or } \mathrm{H}_{2} \\
\text { Aerobic: } \mathrm{S}^{0}\end{array}$ & $\begin{array}{l}+ \\
-\end{array}$ & $\begin{array}{l}\text { Hedrich and Johnson, } \\
\text { 2013a; Johnson et al., } \\
2017\end{array}$ \\
\hline Acidithiobacillus ferriphilus ${ }^{\top}$ & + & + & 2.0 & 30 & Anaerobic: RISC & + & $\begin{array}{l}\text { Falagán and Johnson, } \\
\qquad 2016\end{array}$ \\
\hline Acidithiobacillus ferrivorans ${ }^{\top}$, Peru6 & + & + & $\begin{array}{c}2.5 \\
1.9-3.4\end{array}$ & $\begin{array}{c}27-32 \\
4-37\end{array}$ & $\begin{array}{l}\text { Anaerobic: } S^{0} \\
\text { Aerobic: } S^{0}\end{array}$ & $\begin{array}{l}+ \\
-\end{array}$ & $\begin{array}{l}\text { Hallberg et al., 2010; } \\
\text { Johnson et al., } 2017\end{array}$ \\
\hline Acidithiobacillus ferrooxidans ${ }^{\top}$ & + & + & $\begin{array}{c}2.5 \\
1.3-4.5\end{array}$ & $\begin{array}{l}30-35 \\
10-37\end{array}$ & $\begin{array}{l}\text { Anaerobic: } \mathrm{S}^{0} \\
\text { Aerobic: } \mathrm{S}^{0}\end{array}$ & $\begin{array}{l}- \\
+\end{array}$ & $\begin{array}{c}\text { Pronk et al., 1992; Johnson } \\
\text { et al., } 2017\end{array}$ \\
\hline Acidithiobacillus thiooxidans ${ }^{\top}$ & - & + & $\begin{array}{l}2.0-3.0 \\
0.5-5.5\end{array}$ & $\begin{array}{l}28-30 \\
10-37\end{array}$ & Aerobic: $S^{0}$ & - & Johnson et al., 2017 \\
\hline
\end{tabular}

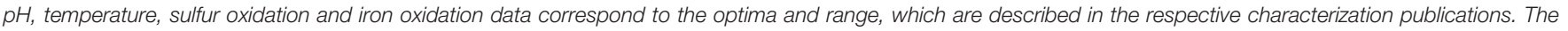
growth column indicates whether dissimilatory iron reduction occurs under the given conditions. Temp, temperature; ox, oxidation; red, reduction; ED, electron donor.

TABLE 4 | Ferric iron-reducing extreme acidophilic mixotrophic bacteria.

\begin{tabular}{|c|c|c|c|c|c|c|c|}
\hline Organism & $\begin{array}{l}\text { Fe } \\
\text { ox. }\end{array}$ & $\begin{array}{l}\mathrm{S}^{0} \\
\mathrm{ox}\end{array}$ & $\mathrm{pH}$ & $\begin{array}{c}\text { Temp. } \\
{\left[{ }^{\circ} \mathbf{C}\right]}\end{array}$ & Conditions Fe red., ED & Growth & References \\
\hline Aciditerrimonas ferrireducens ${ }^{\top}$ & - & ND & $\begin{array}{c}3.0 \\
2.0-4.5\end{array}$ & $\begin{array}{c}50 \\
35-58\end{array}$ & Anaerobic: $\mathrm{H}_{2}$ or $\mathrm{OC}$ & + & Itoh et al., 2011 \\
\hline Sulfobacillus acidophilus $^{\top}$ & + & + & $\sim 2.0$ & $45-50$ & Anaerobic: $\mathrm{S}^{0}$ or $\mathrm{OC}$ & + & $\begin{array}{c}\text { Johnson, 2010; Zhang } \\
\text { et al., } 2021\end{array}$ \\
\hline Sulfobacillus thermosulfidooxidans ${ }^{\top}$ & + & + & $\begin{array}{l}1.7-2.4 \\
1.5-5.5\end{array}$ & $\begin{array}{l}50-55 \\
20-60\end{array}$ & Anaerobic: $\mathrm{H}_{2}, \mathrm{~K}_{2} \mathrm{~S}_{4} \mathrm{O}_{6}$ or $\mathrm{OC}$ & + & $\begin{array}{l}\text { Bridge and Johnson, 1998; } \\
\text { Hedrich and Johnson, } \\
\text { 2013b; Zhang et al., } 2021\end{array}$ \\
\hline
\end{tabular}

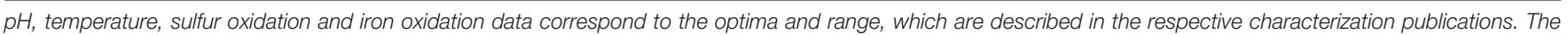

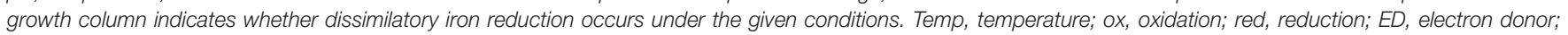
ND, no data; OC, organic carbon.

Falagán et al., 2017). Furthermore, heterotrophic, micro-aerobic Acidicaldus organivorans ${ }^{T}$, Acidobacterium capsulatum $^{\mathrm{T}}$, Acidocella aromatica $^{\mathrm{T}}$, Acidithrix ferrooxidans ${ }^{\mathrm{T}}$, and Sulfolobus acidocaldarius $^{\mathrm{T}}$ cultures showed iron-reducing properties (Johnson et al., 2006; Coupland and Johnson, 2008; Jones et al., 2013; Jones and Johnson, 2015; Masaki et al., 2018). In addition, several micro-aerophilic, acidophilic iron reducers perform this process under anaerobic conditions as well (Falagán and Johnson, 2014; Jones and Johnson, 2015), while others require low oxygen concentrations for this purpose (Falagán et al., 2017). In this context, discriminating between anaerobic, aerobic and micro-aerobic conditions is often difficult, as it is determined by the oxygen solubility under the given conditions. For an accurate classification, the oxygen content would have to be monitored during the entire experiment, which is not always feasible and involves more intricate experimental effort. Accordingly, some of the described aerobic iron reduction processes might actually occur under oxygen limitation, as has been discussed, for instance, for the thermophilic archaeon Sulfolobus acidocaldarius strain 79-13 (Brock and Gustafson, 1976). Therefore, a precise monitoring of the oxygen saturation in the culture medium is essential to obtain information on e.g., inhibition of iron reduction mechanisms by certain oxygen concentrations. This is particularly interesting to understand iron cycling at natural transition zones between oxic and anoxic habitats (Emerson et al., 2010).

\section{Oxygen-Dependent Iron Reduction Processes of the Acidophilic (Chemo-) Organoheterotrophic Genus Acidiphilium}

Comparative investigation on the impact of dissolved oxygen on ferric iron reduction by the extreme acidophile Acidiphilium spp. was conducted (Johnson and Bridge, 2002). Based on their different levels of aerobic iron reduction capacity (Johnson and McGinness, 1991), ferric iron reduction by Acidiphilium acidophilum $^{\mathrm{T}}$ and Acidiphilium sp. SJH was studied under various dissolved oxygen concentrations. Comparison of both, specific iron reduction rates and whole-cell protein profiles of these species, revealed oxygen-dependent differences within the genus Acidiphilium (Johnson and Bridge, 2002). Acidiphilium sp. SJH efficiently reduced ferric iron under aerobic and microaerobic conditions, demonstrated by nearly constant specific 
TABLE 5 | Ferric iron-reducing extreme acidophilic heterotrophic bacteria.

\begin{tabular}{|c|c|c|c|c|c|c|c|}
\hline Organism & $\begin{array}{l}\text { Fe } \\
\text { ox. }\end{array}$ & $\begin{array}{l}S^{0} \\
o x\end{array}$ & $\mathrm{pH}$ & $\begin{array}{l}\text { Temp. } \\
{\left[{ }^{\circ} \mathbf{C}\right]}\end{array}$ & Conditions Fe red., ED & Growth & References \\
\hline Acidicaldus organivorans $^{\top}$ & ND & + & $\begin{array}{c}2.5-3.0 \\
1.75->3.0\end{array}$ & $\begin{array}{l}50-55 \\
40-65\end{array}$ & $\begin{array}{c}\text { Anaerobic: OC } \\
\text { Micro-aerobic: OC }\end{array}$ & $\begin{array}{l}+ \\
+\end{array}$ & Johnson et al., 2006 \\
\hline Acidiferrimicrobium australe ${ }^{\top}$ & + & - & $\begin{array}{c}3.0 \\
1.7-4.5\end{array}$ & $\begin{array}{c}30 \\
20-39\end{array}$ & Anaerobic: OC & + & González et al., 2020 \\
\hline Acidimicrobium ferrooxidans ${ }^{\top}$ & + & - & 2.0 & $45-50$ & Anaerobic: OC & + & Jones and Johnson, 2015 \\
\hline Acidiphilium acidophilum ${ }^{\top}$ & - & + & $\begin{array}{c}2.0 \\
2.0-4.5\end{array}$ & 30 & Micro-aerobic: OC & + & Johnson and Bridge, 2002 \\
\hline $\begin{array}{l}\text { Acidiphilium } \\
\text { sp. SJH }\end{array}$ & ND & ND & 2.0 & 28 & $\begin{array}{c}\text { Aerobic: OC } \\
\text { Micro-aerobic: OC } \\
\text { Anaerobic: OC }\end{array}$ & $\begin{array}{l}+ \\
+ \\
-\end{array}$ & Johnson and Bridge, 2002 \\
\hline Acidithrix ferrooxidans ${ }^{\top}$ & + & - & $2.0-4.4$ & $10-30$ & $\begin{array}{c}\text { Anaerobic: OC } \\
\text { Micro-aerobic: OC }\end{array}$ & $\begin{array}{l}+ \\
+\end{array}$ & Jones and Johnson, 2015 \\
\hline Alicyclobacillus tolerans ${ }^{\top}$ & + & + & $\begin{array}{l}2.5-2.7 \\
1.5-5.0\end{array}$ & $\begin{array}{l}37-42 \\
20-55\end{array}$ & Anaerobic: OC & + & $\begin{array}{l}\text { Karavaiko et al., 2005; } \\
\text { Johnson, } 2010\end{array}$ \\
\hline Ferrimicrobium acidiphilum $^{\top}$ & + & - & $\begin{array}{c}2.0 \\
>1.4\end{array}$ & $\begin{array}{c}35 \\
<37\end{array}$ & Anaerobic: OC & + & Johnson et al., 2009 \\
\hline Ferrithrix thermotolerans $^{\top}$ & + & - & $\begin{array}{c}1.8 \\
>1.6\end{array}$ & $\begin{array}{c}43 \\
<50\end{array}$ & Anaerobic: OC & + & Johnson et al., 2009 \\
\hline Sulfobacillus benefaciens ${ }^{\top}$ & + & + & $\begin{array}{c}1.5 \\
0.8-2.2\end{array}$ & $\begin{array}{c}38.5 \\
30-47\end{array}$ & Anaerobic: OC & + & Johnson et al., 2008 \\
\hline Sulfobacillus harzensis ${ }^{\top}$ & + & + & $\begin{array}{c}3.0 \\
1.5-5.0\end{array}$ & $\begin{array}{c}45 \\
25-55\end{array}$ & Anaerobic: OC & + & Zhang et al., 2021 \\
\hline Sulfobacillus sibiricus ${ }^{\top}$ & + & + & $\begin{array}{c}2.0 \\
1.1-2.6\end{array}$ & $\begin{array}{c}55 \\
17-60\end{array}$ & Anaerobic: OC & + & Zhang et al., 2021 \\
\hline Sulfobacillus thermotolerans ${ }^{\top}$ & + & + & $\begin{array}{c}2.0 \\
1.2-2.4\end{array}$ & $\begin{array}{c}40 \\
20-60\end{array}$ & Anaerobic: OC & + & Zhang et al., 2021 \\
\hline
\end{tabular}

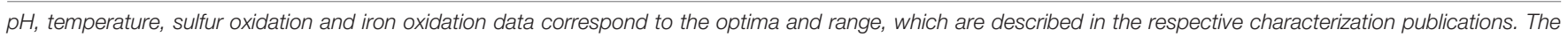

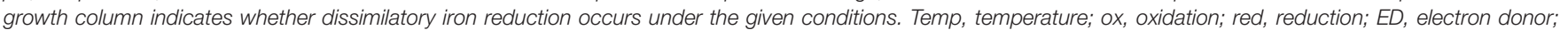
ND, no data; OC, organic carbon.

iron reduction rates between 40 and $80 \%$ dissolved oxygen. In contrast, effective iron reduction by Acidiphilium acidophilum ${ }^{\mathrm{T}}$ was only observed under micro-aerobic conditions (20-40\% dissolved oxygen). Both strains did not reduce iron in the absence of oxygen. The detection of three additional proteins in Acidiphilium acidophilum ${ }^{\mathrm{T}}$ under micro-aerobic conditions indicated an oxygen-inducible iron reduction mechanism for this species. Since the dissolved oxygen content had no significant influence on the protein profile of Acidiphilium SJH, it was suggested to feature a constitutive iron reductase system. This tendency was also detected in further species of Acidiphilium, dividing the genus into two groups (Johnson and Bridge, 2002). However, Acidiphilium cryptum JF-5 reduced ferric iron independent of the given oxygen saturation even under anaerobic conditions at pH 3 (Küsel et al., 2002). Still, small amounts of oxygen are required to support growth of Acidiphilium species (Johnson and Bridge, 2002).

\section{Aerobic Iron Reduction at Extremely Low pH}

In oxygen-rich environments, the detection of ferric iron reduction is hindered by the auto- and microbial oxidation of the generated ferrous iron. Consequently, the study of iron reduction processes under these conditions is challenging. The higher stability of ferrous iron at low $\mathrm{pH}$ values enables the monitoring of ferric iron reduction even in the presence of oxygen. Already in the 1970s, aerobic ferrous iron formation was reported for sulfur-oxidizing Acidithiobacillus thiooxidans and Acidithiobacillus ferrooxidans at low pH (Brock and Gustafson, 1976; Sand, 1989). Further, iron oxidation of At. ferrooxidans was non-detectable under these extremely acidic conditions. This led to the assumption that the iron oxidation chain of this species is inhibited below $\mathrm{pH} 1.3$, preventing re-oxidation of the ferrous iron formed. A pH increase to 1.8 again enabled rapid ferrous iron oxidation (Sand, 1989). These results contradicted the until then prevailing assumption of exclusively anaerobic iron reduction by At. ferrooxidans (Brock and Gustafson, 1976). Also, strains belonging to the acidophilic, heterotrophic genus of Acidiphilium, were characterized as aerobic iron reducers, using glucose or glycerol as substrate (Johnson and McGinness, 1991). When comparing iron reduction rates, which are often used to obtain tendencies of organisms to reduce iron under certain conditions, it is important to respect what the numbers are referring to. In the case of Acidiphilium cryptum JF-5, the non-normalized ferric iron reduction rate of the anaerobic experiment $\left(24.2 \mathrm{mmol} \mathrm{L}^{-1}\right.$ day $\left.^{-1}\right)$ was smaller than at oxygen saturation $\left(46.6 \mathrm{mmol} \mathrm{L}^{-1}\right.$ day $\left.^{-1}\right)$, while normalizing the rate to cell density showed a lower iron reduction rate at oxygen saturation, which was attributed to 


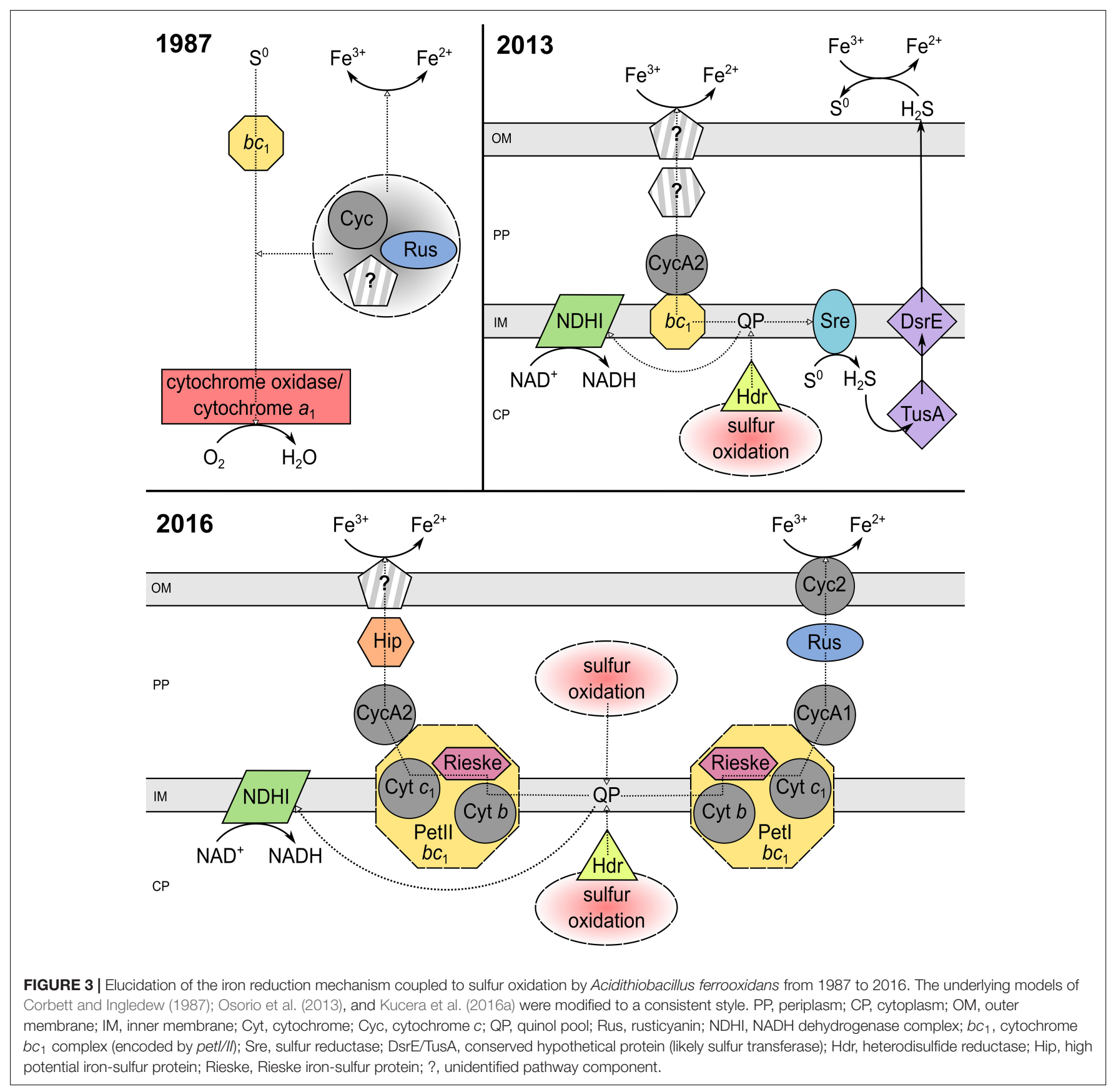

better growth under these conditions (Küsel et al., 2002). Despite that, aerobic iron reduction was growth-related in further species, like Acidithiobacillus thiooxidans, Acidiphilium cryptum, and Acidiphilium sp. SJH (Brock and Gustafson, 1976; Johnson and McGinness, 1991). However, in Sulfolobus acidocaldarius and At. ferrooxidans, accumulation of ferrous iron was not accompanied by cell growth (Brock and Gustafson, 1976; Sand, 1989). Therefore, it remained unclear whether this was a microbial respiratory process or a non-enzymatic, chemical reduction mediated by metabolic intermediates accumulated in the culture liquor. Additionally, abiotic ferric iron reduction was shown for uninoculated flasks at thermophilic conditions $\left(70^{\circ} \mathrm{C}\right)$. Still, the amount of iron reduced in inoculated batches was considerably higher (Brock and Gustafson, 1976).

\section{Insights Into the Aerobic Ferric Iron Reduction Mechanisms in Acidithiobacilli}

In recent years, aerobic ferric iron reduction ability was reported for various Acidithiobacillus species (Table 3) reducing soluble ferric iron and/or mediating the reductive dissolution of ferric iron ores (Marrero et al., 2015, 2017; Johnson et al., 2017; Smith and Johnson, 2018). The results obtained from these studies indicate species-dependent mechanisms for the "latent iron reduction" by acidithiobacilli (Johnson et al., 2021). Thereby, a 


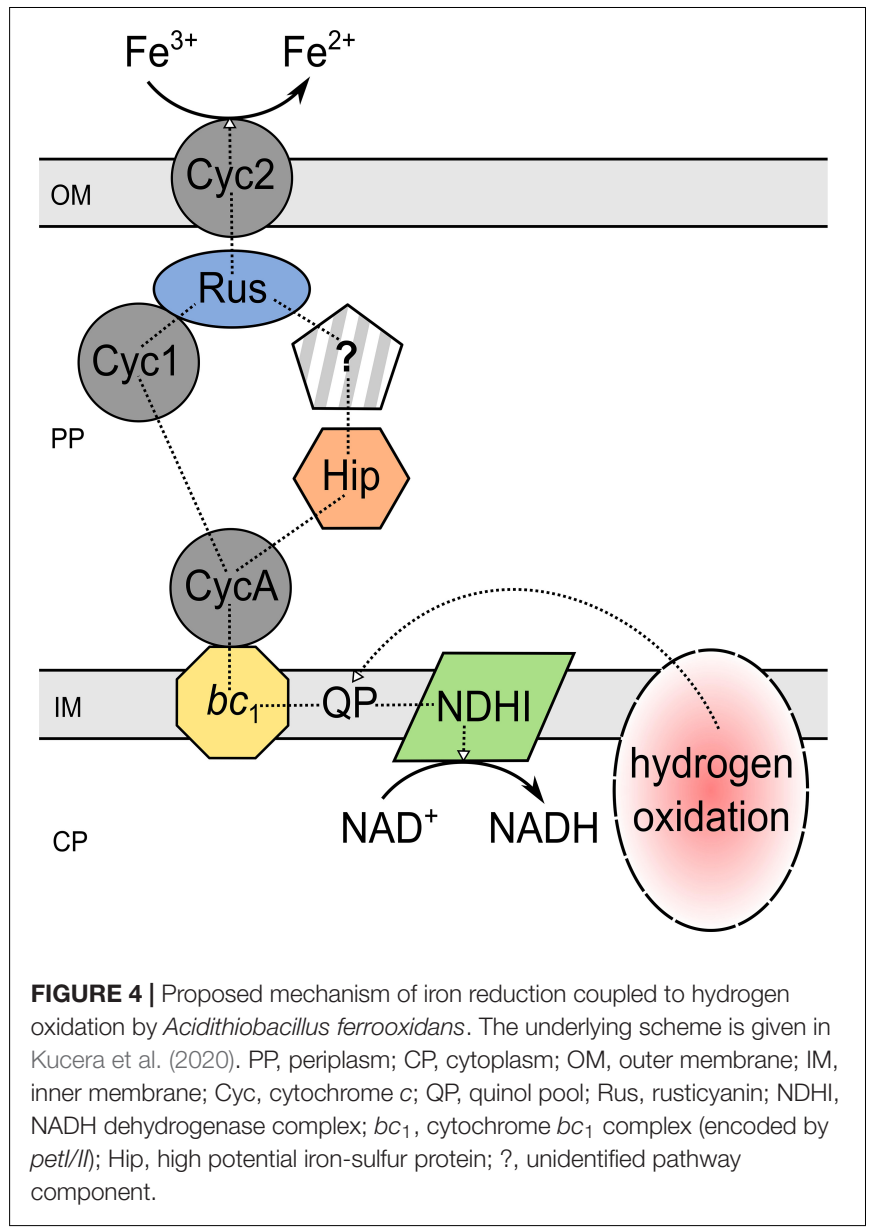

non-growth-related mechanism was assumed for At. thiooxidans (Marrero et al., 2015), which contradicted the formerly proposed correlation between ferrous iron accumulation and cell growth in cultures of At. thiooxidans (Brock and Gustafson, 1976). Furthermore, cell-free supernatants ( $\mathrm{pH} 1.0)$ of sulfur-respiring At. caldus and At. ferridurans, also showed aerobic iron reduction, indicating a cell-independent chemical reduction process (Johnson et al., 2017). However, in the case of At. thiooxidans, washed cells were also capable of reducing iron within a short time under aerobic conditions $(150-200 \mathrm{mg} / \mathrm{L}$ in $6.5 \mathrm{~h})$ regardless of the $\mathrm{pH}(0.9,2.5,3.5)$ (Marrero et al., 2015). Initially, the aerobic iron reduction observed in Acidithiobacillus (especially At. thiooxidans) cultures was exclusively assigned to the reduction of ferric iron by sulfur intermediates formed during sulfur respiration under aerobic conditions (Marrero et al., 2015, 2017). However, Johnson et al. (2017, 2021) provided evidence, that aerobic ferric iron reduction mediated by acidithiobacilli is not only attributed to sulfur oxidation, as hydrogen-grown At. caldus and tetrathionate- or hydrogen-metabolizing At. thiooxidans reduced ferric iron under these conditions as well. Recently, indications were given that an initial hydrophilization of sulfur by acidithiobacilli may enable chemical, ferric iron reduction as tests with bio-activated, heat-sterilized sulfur allowed aerobic iron reduction at $50^{\circ} \mathrm{C}$ and $\mathrm{pH} 0.9-1.0$ as well (Johnson et al., 2021). These finding may also explain the $\mathrm{pH}$-independent iron reduction of washed At. thiooxidans cells by cell-surface-associated activated sulfur particles. Accordingly, further studies are required to gain more insight into the processes that occur during aerobic iron reduction at low $\mathrm{pH}$. Moreover, the aforementioned aerobic reduction of ferric iron by heterotrophic Acidiphilum species (Johnson and McGinness, 1991) implies that there are several mechanisms enabling ferrous iron formation in the presence of oxygen. Whether these mechanisms are enzymatic, non-enzymatic, or a combination of both should be further investigated. In this context, the detection and identification of intermediates of the sulfur metabolism is a major drawback due to their low stability and rapid degradation (Rohwerder, 2002).

\section{APPLICATION AND ENVIRONMENTAL ASPECTS}

Reductive dissolution by acidophiles was shown for several ferric iron-containing minerals, as ferric hydroxide $\left[\mathrm{Fe}(\mathrm{OH})_{3}\right]$, goethite $(\alpha-\mathrm{FeOOH})$, akageneite $(\beta-\mathrm{FeOOH})$, magnetite $\left(\mathrm{Fe}_{3} \mathrm{O}_{4}\right)$, potassium jarosite $\left[\mathrm{KFe}_{2}\left(\mathrm{SO}_{4}\right)_{2}(\mathrm{OH})_{6}\right]$, and natrojarosite $\left[\mathrm{NaFe}_{2}\left(\mathrm{SO}_{4}\right)_{2}(\mathrm{OH})_{6}\right]$ (Bridge and Johnson, 1998, 2000). These findings opened new possibilities for biohydrometallurgical processes where oxidative bioleaching is not effective or more sustainable methods should be established. Nevertheless, as other metal recovery methods, anaerobic (AnRD) and aerobic reductive dissolution (AeRD) require special operating conditions which have to be considered especially regarding operational costs. The maintenance of an anaerobic process requires a closed system and exclusion of oxygen, e.g., by supply of nitrogen. Further, AnRD is an acid-consuming reaction involving the addition of acid (Marrero et al., 2015), while AeRD can operate as net acid-producing process (Johnson et al., 2021). On the downside, the extremely low pH during AeRD is only tolerated by few microorganisms, which minimizes the number of suitable acidophiles. Moreover, the exclusion of aerobic iron oxidizers from AeRD applications may require an anaerobic phase integrated into the process to avoid interference with iron-oxidizing leptospirilli (Marrero et al., 2015). Still, reductive bioleaching is a promising method to exploit even low-grade ferric iron minerals and to recycle process wastes of traditional hydrometallurgical methods, e.g., laterite tailings (Marrero et al., 2015).

\section{Biohydrometallurgical Application of Microbial Iron Reduction}

The success of biotechnological applications in the metallurgical sector is evident from copper production via bioleaching in e.g., Chile and elsewhere in the world (Gentina and Acevedo, 2016). Despite the fact that biohydrometallurgical processes are nowadays well established, commercial applications are still limited to sulfidic minerals (Johnson, 2014). In the case of oxidative metal dissolution by acidophiles, the process is enabled by the sulfuric acid produced during sulfur oxidation by the microorganisms and/or the ferric iron generated by 
microbial iron oxidation of the mineral (Johnson et al., 2013). The mechanism underlying reductive dissolution of ferric iron minerals is also caused, to some extent, by acidolysis (Marrero et al., 2015). Further, an equilibrium shift between iron in the solid phase and in solution created by microbial ferric iron reduction accelerates mineral dissolution (Bridge and Johnson, 1998). Although further research is required to understand the exact mechanisms involved, reductive bioleaching is already enabling new, economic recovery methods for different ferric iron-associated metals in laboratory scale.

\section{Processing Ferric Iron Ores for Base Metal Recovery}

Over the last decade, a lot of research has been conducted on the application of reductive microbial dissolution processes focusing on the extraction of metals from limonitic laterites (Table 6). Approximately $70 \%$ of world's land-based nickel resources are present in the form of laterite (Dalvi et al., 2004). Laterites are commercially processed by reagent- and energydemanding pyrometallurgical (smelting) or hydrometallurgical (CARON process or high pressure acid leaching) processes (Dalvi et al., 2004). Nickel laterites consist of two zones, the upper limonite and the lower saprolite zone, of which the limonite one contains base metals associated with ferric iron in the form of modified goethite (Johnson et al., 2013). Bioleaching of limonite by acidithiobacilli is achieved through microbial acid generation, lowering the redox potential under anaerobic conditions, iron reduction under aerobic conditions and activating/"wetting" of naturally hydrophobic sulfur (Johnson et al., 2021). Advantages compared to conventional pyroand hydrometallurgical methods are cost reduction by using inorganic energy sources (e.g., sulfur) and an ambient operating temperature (Johnson et al., 2013). The extremely low pH during AeRD and AnRD enhances the acidic attack during mineral dissolution and reduces metal precipitation, thereby simplifying downstream processing which is a major advantage compared to neutrophilic iron reduction approaches (Johnson et al., 2013).

According to the diverse composition of laterites, different base metals have already been the target of reductive bioleaching, such as nickel (Hallberg et al., 2011a), copper (Nancucheo et al., 2014), and cobalt (Smith et al., 2017), reaching up to $100 \%$ yield. It also became apparent, that the geographical origin of the ore and its mineralogical composition had a major impact on their biohydrometallurgical application (Santos et al., 2020). Initially, the applicability of reductive bioleaching was examined by using pure cultures of Acidithiobacillus ferrooxidans operating under anoxic conditions (Hallberg et al., 2011a; Johnson et al., 2013; Nancucheo et al., 2014). Afterward, other acidophiles and consortia as well as different aeration setups (AeRD, AeAnRD and AnRD) were investigated for this reductive extraction approach (Marrero et al., 2015; Santos et al., 2020; Johnson et al., 2021). In some processes, also thermotolerant strains were used (Smith et al., 2017; Johnson et al., 2021) which enable higher operation temperatures and thereby enhanced reaction kinetics. However, the use of consortia also showed that the diversity of the microbial community decreases during the reductive bioleaching process, which could sometimes be due to a lack of adaptation of the acidophiles to the mineral, resulting in the predominance of a few strains (Smith et al., 2017; Santos et al., 2020). Monitoring of cell numbers by qPCR also showed a constant number of active bacteria during the AeRD process and a decrease after 3 days in the case of AnRD and Ae-AnRD (Marrero et al., 2015). Moreover, comparison of reductive limonite dissolution by a mesophilic consortium of At. ferrooxidans ${ }^{\mathrm{T}}$, At. ferriphilus ${ }^{\mathrm{T}}$, At. ferridurans ${ }^{\mathrm{T}}$, Sulfobacillus acidophilus ${ }^{\mathrm{T}}$, and Sulfobacillus thermosulfidooxidans ${ }^{\mathrm{T}}$ showed higher base metal yields in the anaerobic bioreactor, while the aerobic bioreactor required no further acid supply after 9 days (Johnson et al., 2021). Smith et al. (2017) observed the same tendency for cobalt extraction from limonite, which was more efficient using the anaerobic reductive leaching approach and seemed to correlate with manganese dissolution. In contrast, AeRD experiments with pure cultures of non-iron-oxidizing acidithiobacilli (At. thiooxidans or At. caldus) resulted in higher metal yields compared to AnRD approaches with the same limonite (Marrero et al., 2017; Johnson et al., 2021). Marrero et al. (2017) provided a direct comparison of AnRD and AeRD of nickel laterite overburden by At. ferrooxidans or At. thiooxidans, respectively, showing approximately the same yields. Accordingly, in this case AeRD was selected as the favorable method since it does not require anoxic conditions, it enables better extraction kinetics, and the extremely low $\mathrm{pH}$ reduces iron precipitation (Marrero et al., 2017). The success of bioprocessing of limonitic ores depends on the mineralogy. If large amounts of acid-unstable ferrous iron minerals are present, manganese-associated cobalt is readily solubilized by microbial acid generation under aerobic conditions, whereas ferric iron reduction by acidophiles promotes solubilization of ferric iron minerals and nickel (Johnson et al., 2021). In addition, microbial ferrous iron generation accelerates the reductive dissolution on Mn (IV) minerals (Santos et al., 2020; Johnson and Pakostova, 2021).

All this indicates that, as in any biotechnological process, a large number of parameters need to be optimized to obtain the best possible results. These are highly influenced by the mineral composition, the bioleaching method and the microorganisms involved. Accordingly, pre-investigations concerning the characteristics of the applied acidophiles and ores are crucial. Furthermore, abiotic effects during reductive bioleaching must be considered. In the context of abiotic effects caused by chemical leaching via acid dissolution at extremely low $\mathrm{pH}$ values used for AnRD and AeRD, it was shown that bacterial catalyzed approaches with iron reducers allow more effective metal dissolution than acidolysis alone (Nancucheo et al., 2014). Reductive bioleaching was also up to 6 times more effective than acid leaching in processing cobalt-containing limonite from Kazakhstan or the Philippines under aerobic conditions (Smith et al., 2017). Further benefits of reductive bioleaching approaches were shown by an initial anaerobic phase during leaching of polymetallic sulfides which had to some extend positive effects on the base metal recovery and enhanced the iron removal noticeable at both 45 and $70^{\circ} \mathrm{C}$ (Norris et al., 2015).

Additional to the bioleaching of base metals from laterites, iron reduction by acidophiles offers far more application potential. The bio-reductive processes would be especially suitable to recycle scrap electronics or bioremediate 
TABLE 6 | Overview of reductive mineral dissolution using extreme acidophiles.

\begin{tabular}{|c|c|c|c|c|c|}
\hline Mineral & Origin & Microorganisms & Method & Yield & References \\
\hline Nickel laterite & Western Australia & At. ferrooxidans ${ }^{\top}$ & $A n R D$ & $>80 \% \mathrm{Ni}$ & Hallberg et al., 2011a \\
\hline Nickel laterite & Western Australia & At. ferrooxidans ${ }^{\top}$ & AeRD & $\sim 10 \% \mathrm{Ni}$ & Hallberg et al., 2011a \\
\hline Limonitic nickel laterite & Australia & At. ferrooxidans ${ }^{\top}$ & $A n R D$ & $\begin{array}{l}46-82 \% \mathrm{Ni} \\
64-90 \% \\
\mathrm{Co} \\
75-116 \% \\
\mathrm{Mn}\end{array}$ & Johnson et al., 2013 \\
\hline Copper laterite & Carajás Belt, Pará State, Brazil & At. ferrooxidans ${ }^{\top}$ & $A n R D$ & $\leq 78 \% \mathrm{Cu}$ & Nancucheo et al., 2014 \\
\hline Laterite tailings & $\begin{array}{l}\text { Moa, Cuba; from CARON } \\
\text { process }\end{array}$ & At. thiooxidans ${ }^{\top}$ & AeRD & $\begin{array}{l}53 \% \mathrm{Ni} \\
46 \% \mathrm{Co}\end{array}$ & Marrero et al., 2015 \\
\hline Laterite tailings & $\begin{array}{l}\text { Moa, Cuba; from CARON } \\
\text { process }\end{array}$ & $\begin{array}{l}\text { At. thiooxidans }{ }^{\top} \text { and } A t . \\
\text { ferrooxidans }\end{array}$ & AeRD & $\begin{array}{l}56 \% \mathrm{Ni} \\
60 \% \mathrm{Co}\end{array}$ & Marrero et al., 2015 \\
\hline Laterite tailings & $\begin{array}{l}\text { Moa, Cuba; from CARON } \\
\text { process }\end{array}$ & $\begin{array}{l}\text { At. thiooxidans }{ }^{\top} \text { and } A t \text {. } \\
\text { ferrooxidans }^{\top}\end{array}$ & $\mathrm{Ae}-\mathrm{AnRD}$ & $\begin{array}{l}53 \% \mathrm{Ni} \\
58 \% \mathrm{Co}\end{array}$ & Marrero et al., 2015 \\
\hline Laterite tailings & $\begin{array}{l}\text { Moa, Cuba; from CARON } \\
\text { process }\end{array}$ & At. ferrooxidans ${ }^{\top}$ & AnRD & $\begin{array}{l}58 \% \mathrm{Ni} \\
56 \% \mathrm{Co}\end{array}$ & Marrero et al., 2015 \\
\hline Nickel laterite overburden & Punta Gorda, Moa, Cuba & At. thiooxidans ${ }^{\top}$ & AeRD & $\begin{array}{l}16 \% \mathrm{Ni} \\
85 \% \mathrm{Co} \\
74 \% \mathrm{Mn}\end{array}$ & Marrero et al., 2017 \\
\hline Nickel laterite overburden & Punta Gorda, Moa, Cuba & At. ferrooxidans ${ }^{\top}$ & AnRD & $\begin{array}{l}16 \% \mathrm{Ni} \\
78 \% \mathrm{Co} \\
86 \% \mathrm{Mn}\end{array}$ & Marrero et al., 2017 \\
\hline $\begin{array}{l}\text { Cobalt-bearing limonitic laterite } \\
\text { ore }\end{array}$ & Shevchenko, Kazakhstan & $\begin{array}{l}\text { Consortium mesophilic and } \\
\text { thermotolerant bacteria }{ }^{a}\end{array}$ & AnRD & $\begin{array}{l}50-70 \% \mathrm{Ni} \\
89-99 \% \\
\text { Co }\end{array}$ & Smith et al., 2017 \\
\hline $\begin{array}{l}\text { Cobalt-bearing limonitic laterite } \\
\text { ore }\end{array}$ & Acoje mine, Philippines & $\begin{array}{l}\text { Consortium mesophilic and } \\
\text { thermotolerant bacteria }\end{array}$ & AnRD & $\begin{array}{l}40 \% \mathrm{Ni} \\
90 \% \mathrm{Co}\end{array}$ & Smith et al., 2017 \\
\hline Cobalt-bearing limonitic materials & $\begin{array}{l}\text { Kastoria, Agios loannis and Evia } \\
\text { mine, Greece }\end{array}$ & $\begin{array}{l}\text { Consortium mesophilic } \\
\text { bacteria }^{b}\end{array}$ & AnRD & $\begin{array}{l}37-73 \% \mathrm{Ni} \\
40-50 \% \\
\mathrm{Co} \\
15-52 \% \\
\mathrm{Mn}\end{array}$ & Santos et al., 2020 \\
\hline Limonite ore & Shevchenko, Kazakhstan & $\begin{array}{l}\text { Consortium mesophilic } \\
\text { bacteria }^{c}\end{array}$ & AnRD & $\begin{array}{l}\sim 55 \% \mathrm{Ni} \\
\sim 90 \% \mathrm{Co} \\
\sim 95 \% \mathrm{Mn}\end{array}$ & Johnson et al., 2021 \\
\hline Limonite ore & Shevchenko, Kazakhstan & $\begin{array}{l}\text { Consortium mesophilic } \\
\text { bacteria }^{c}\end{array}$ & AeRD & $\begin{array}{l}\sim 25 \% \mathrm{Ni} \\
\sim 20 \% \mathrm{Co} \\
\sim 40 \% \mathrm{Mn}\end{array}$ & Johnson et al., 2021 \\
\hline Limonite ore & Shevchenko, Kazakhstan & At. caldus ${ }^{\top}$ & AeRD & $\begin{array}{l}\sim 100 \% \mathrm{Ni} \\
\sim 95 \% \mathrm{Co} \\
\sim 90 \% \mathrm{Mn}\end{array}$ & Johnson et al., 2021 \\
\hline
\end{tabular}

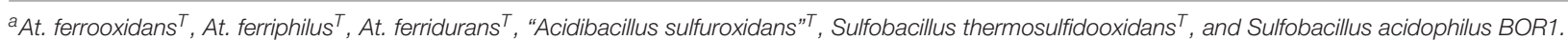

${ }^{b}$ At. ferrooxidans ${ }^{T}$, At. ferrooxidans CF3, At. ferriphilus ${ }^{T}$, At. ferridurans ${ }^{T}$, and Sulfobacillus thermosulfidooxidans ${ }^{T}$.

${ }^{c}$ At. ferrooxidans ${ }^{T}$, At. ferriphilus ${ }^{T}$, At. ferridurans ${ }^{T}$, Sulfobacillus acidophilus ${ }^{T}$, and Sulfobacillus thermosulfidooxidans ${ }^{T}$.

$A n R D$, anaerobic reductive dissolution; AeRD, aerobic reductive dissolution; Ae-AnRD, aerobic reductive dissolution with an integrated anaerobic phase.

metal-contaminated soil (Johnson et al., 2013). Another approach aimed at the pretreatment of laterite-associated monazite, a rare earth elements-containing phosphate mineral, by ferric iron reduction to improve the monazite exposure for further acid dissolution (Nancucheo et al., 2019). However, due to the innovation of this method, further research is required before it is ready for application.

\section{Introducing a Reductive Bioleaching Circuit: The Ferredox Concept}

In 2011, a generic concept for ferric iron mineral processing, Ferredox, was presented for tropical limonitic laterite (du Plessis et al., 2011). In assembly, the model has not yet been applied in a full-scale operation, but it has been proposed as a promising sustainable metal recovery method. The modular process relies on the findings that anaerobic reductive dissolution of goethite can take place under ambient conditions utilizing bacterial catalysis (Hallberg et al., 2011a). This new perspective creates a biohydrometallurgical method, which might allow iron oxide treatment with reduced material and processing costs. Overall, the process consists of four modularized main stages: reductive bioleaching, metal recovery, iron oxidation/precipitation, and reductive acid generation. During the initial reductive bioleaching, iron-oxidizing/reducing 
acidithiobacilli couple the oxidation of elemental sulfur to the reduction of ferric iron present in goethite and other minerals, solubilizing target metals. The resulting pregnant leaching solution is subsequently processed to extract the focused base metals. After a two staged iron oxidation process, the formed iron precipitates are utilized to generate the sulfuric acid consumed during the initial reductive dissolution of goethite $\left[\mathrm{S}^{0}+6 \mathrm{FeO}(\mathrm{OH})+10 \mathrm{H}^{+} \rightarrow \mathrm{SO}_{4}^{2-}+6 \mathrm{Fe}^{2+}+8 \mathrm{H}_{2} \mathrm{O}\right]$. In turn, this process will make use of the metabolism of At. ferrooxidans once again by anaerobic oxidation of elemental sulfur generating acid $\left(\mathrm{S}^{0}+6 \mathrm{Fe}^{3+}+4 \mathrm{H}_{2} \mathrm{O} \rightarrow \mathrm{SO}_{4}{ }^{2-}+6 \mathrm{Fe}^{2+}+8 \mathrm{H}^{+}\right)$. Advantages of the given Ferredox concept are biomass recycling and enhancement due to the use of At. ferrooxidans in multiple process steps and increased sustainability due to biologically catalyzed reactions and operation at ambient conditions (du Plessis et al., 2011; Johnson and du Plessis, 2015).

\section{Environmental Impact of Acidophilic Iron Reducers}

In an ancient, oxygen-free atmosphere, the usage of ferric iron as electron acceptor may have had a major role and impact on live (Walker, 1987; Slobodkin, 2005). Currently, oxido-reduction of iron has been demonstrated for both pure and mixed cultures of acidophiles (Johnson et al., 1993). It is now known that microbial iron cycling effects inorganic and organic contaminants, carbon cycling, and nutrient mobilization in the environment (Kappler and Straub, 2005). For instance, iron-reducing acidophiles were shown to eliminate the toxicity of some oxy-anions through ferrous iron generation, e.g., the more toxic $\mathrm{Cr}(\mathrm{VI})$ (hydrogen chromate, $\mathrm{HCrO}_{4}{ }^{-}$) is reduced to the less harmful $\mathrm{Cr}$ (III) by ferrous iron (Johnson et al., 2017).

Again, the dissolution of iron oxides releases metal cations absorbed to the mineral, which may have a negative impact on the biosphere (Kappler and Straub, 2005). Nevertheless, ferrous iron, the product of ferric iron reduction, can in turn act as a strong reductant for organic pollutants, e.g., nitroaromatics (Hofstetter et al., 1999).

The fact that acidophilic iron reducers feature autotrophic as well as heterotrophic metabolisms ensures their function as both, producers and degraders in ecosystems (Kashefi et al., 2004). The resulting complete mineralization of organic matter prevents accumulation of high amounts of organic substances which could have a toxic effect, e.g., small organic acids, on autotrophic acidophilic microorganisms. In acid streamers, dead organic matter and generated iron precipitates settle down to anoxic, organotrophic acidophiles-inhabitated zones, which were shown to feature more ferrous than ferric iron due to the occurring oxidation of organic substances coupled to iron reduction (Johnson et al., 1993, 2012). Thus, acidophiles actively contribute to the carbon and iron cycle. In the river Rio Tinto, iron transformation was shown to be mainly enabled by the interaction of the autotrophic iron oxidizers Leptospirillum ferrooxidans and At. ferrooxidans in aerobic areas and iron reduction by At. ferrooxidans (autotrophic) and Acidiphilium species (heterotrophic) in anaerobic zones (González-Toril et al., 2003). Investigations of the microbial diversity in sediments of an acidic lignite mine lake (Lusatia, Germany) revealed further iron reducers and cycling in these environments. Isolates closest related to species of Acidiphilium, Acidocella, Ferrimicrobium, Acidithiobacillus, Alicyclobacillus, and Acidobacteriaceae reduced iron in this habitat, with some of them being found throughout all zones of the sediment ( $\mathrm{Lu}$ et al., 2010). Likewise, previous investigations of the same coal mine lake emphasized this $\mathrm{pH}$ gradient-dependent heterogeneity of ferric iron-reducing acidophiles in the sediment (Blöthe et al., 2008). In addition, two of the isolated Alicyclobacillus relatives showed iron cycling capacity in the laboratory by dissolving schwertmannite and re-oxidizing the ferrous iron by forming schwertmannite precipitates again (Lu et al., 2010). Still, the capacity of iron cycling by pure cultures was previously described for other acidophilic isolates (Johnson et al., 1993). Nevertheless, in many acidic ecosystems, a diverse community of phyla is responsible for the conversion of iron and other elements. Depending on the conditions, a closed iron cycle can be independently realized by individual species. According to the manifold influences of acidophilic iron reducers on different ecosystems and material cycles, it is important to study this group of microorganisms and thus to achieve a better awareness of human interventions, especially with regard to anthropogenically induced acidic and metal-rich habitats.

\section{CONCLUSION AND PERSPECTIVES}

Although iron reduction processes mediated by acidophiles have been known for nearly 50 years, it is only in the last decade that much interest has developed in their application. This especially included the utilization of well-studied ferric iron reducers of the genus Acidithiobacillus. Nevertheless, far more iron-reducing acidophiles have been identified including over 20 different genera. This is justified by the growing impact of this metabolic trait due to the recently known suitability for reductive bioleaching of ferric iron minerals. Accordingly, the characterization of novel acidophilic microorganisms now usually includes testing for iron reduction, which was not necessarily the case in the past. Still, few of the newly discovered iron reducers are investigated in detail. This lack of knowledge offers a great demand for further research particularly with regards to thermophilic conditions which could enhance the leaching kinetics. Moreover, the different mechanisms of ferric iron reduction in acidophiles (anaerobic, micro-aerobic or aerobic) allow diverse operation setups for base metal processing from laterites and a correspondingly higher freedom to customize the metal extraction method according to the mineral composition. Recently, the high potential to use acidophile-promoted reductive dissolution was demonstrated by exceeding base metal extraction from ferric minerals and overburden. Approaches were applied to recycle low-grade wastes from traditional laterite processing by reductive bioleaching. Additionally, first approaches to pretreat rare earthbearing minerals associated with laterites showed promising results. Besides, ferric iron reduction by acidophiles might further be suitable for bioremediation and scrap electronics recycling. 
All the benefits of such an innovative application profit from precise knowledge of the underlying microbiological processes and mineral composition. Both call for suitable devices and expertise in microbiological and mineralogical fields which may in some cases rely on an interdisciplinary cooperation. Further adjustments of this biohydrometallurgical approach require a sufficient understanding of the biochemical iron reduction mechanism, especially concerning acidophiles apart from acidithiobacilli. As Sulfobacillus thermosulfidooxidans was shown to be the predominant microorganism (beside Acidithiobacillus ferrooxidans) in reductive bioleaching experiments with moderate consortia, this acidophile seems to be an outstanding candidate for detailed investigations of its iron reduction mechanism. The biochemical information obtained for At. ferrooxidans offered new knowledge and interesting insights, by

\section{REFERENCES}

Ai, C., Liang, Y., Miao, B., Chen, M., Zeng, W., and Qiu, G. (2018). Identification and analysis of a novel gene cluster involves in $\mathrm{Fe}^{2+}$ oxidation in Acidithiobacillus ferrooxidans ATCC 23270, a typical biomining acidophile. Curr. Microbiol. 75, 818-826. doi: 10.1007/s00284-018-1 453-9

Amouric, A., Brochier-Armanet, C., Johnson, D. B., Bonnefoy, V., and Hallberg, K. B. (2011). Phylogenetic and genetic variation among $\mathrm{Fe}(\mathrm{II})$-oxidizing acidithiobacilli supports the view that these comprise multiple species with different ferrous iron oxidation pathways. Microbiology 157, 111-122. doi: 10. 1099/mic.0.044537-0

Auernik, K. S., and Kelly, R. M. (2010). Impact of molecular hydrogen on chalcopyrite bioleaching by the extremely thermoacidophilic archaeon Metallosphaera sedula. Appl. Environ. Microbiol. 76, 2668-2672. doi: 10.1128/ AEM.02016-09

Blöthe, M., Akob, D. M., Kostka, J. E., Göschel, K., Drake, H. L., and Küsel, K. (2008). pH gradient-induced heterogeneity of Fe(III)-reducing microorganisms in coal mining-associated lake sediments. Appl. Environ. Microbiol. 74, 10191029. doi: 10.1128/AEM.01194-07

Bonnefoy, V. (2010). "Bioinformatics and genomics of iron- and sulfur-oxidizing acidophiles," in Geomicrobiology: Molecular and Environmental Perspective, eds L. L. Barton, M. Mandl, and A. Loy (Dordrecht: Springer), 169-192.

Bridge, T. A. M., and Johnson, D. B. (1998). Reduction of soluble iron and reductive dissolution of ferric iron-containing minerals by moderately thermophilic ironoxidizing bacteria. Appl. Environ. Microbiol. 64, 2181-2186. doi: 10.1128/AEM. 64.6.2181-2186.1998

Bridge, T. A. M., and Johnson, D. B. (2000). Reductive dissolution of ferric iron minerals by Acidiphilium SJH. Geomicrobiol. J. 17, 193-206. doi: 10.1080/ 01490450050121161

Brock, T. D., and Gustafson, J. (1976). Ferric iron reduction by sulfur- and ironoxidizing bacteria. Appl. Environ. Microbiol. 32, 567-571. doi: 10.1128/AEM. 32.4.567-571.1976

Bromfield, S. M. (1954). The reduction of iron oxide by bacteria. J. Soil Sci. 5, 129-139. doi: 10.1111/j.1365-2389.1954.tb02181.x

Corbett, C. M., and Ingledew, W. J. (1987). Is $\mathrm{Fe}^{3+/ 2+}$ cycling an intermediate in sulphur oxidation by $\mathrm{Fe}^{2+}$-grown Thiobacillus ferrooxidans. FEMS Microbiol. Lett. 41, 1-6. doi: 10.1111/j.1574-6968.1987.tb02131.x

Coupland, K., and Johnson, D. B. (2008). Evidence that the potential for dissimilatory ferric iron reduction is widespread among acidophilic heterotrophic bacteria. FEMS Microbiol. Lett. 279, 30-35. doi: 10.1111/j.15746968.2007.00998.x

Dahl, C. (2020). "A biochemical view on the biological sulfur cycle," in Environmental Technologies to Treat Sulphur Pollution: Principles and Engineering, ed. P. N. L. Lens (London: IWA Publishing), 55-96.

Dalvi, A. D., Bacon, W. G., and Osborne, R. C. (eds) (2004). "The past and the future of nickel laterites," in Proceedings of the PDAC 2004 International Conference Trade Show and Investors Exchange, Toronto, 7-10. showing a reverse usage of iron oxidation chain components. Accordingly, a comprehensive insight into the iron reduction mechanisms of other acidophiles, especially those with iron oxidation pathways other than At. ferrooxidans, would be of great interest. The research field of reductive bioleaching would benefit from further studies on additional acidophilic genera and even archaea.

\section{AUTHOR CONTRIBUTIONS}

LM: writing manuscript, preparing figures, and tables. SH: conceptual discussions, revising, and editing manuscript. Both authors contributed to the article and approved the submitted version.

Das, A., Mishra, A. K., and Roy, P. (1992). Anaerobic growth on elemental sulfur using dissimilar iron reduction by autotrophic Thiobacillus ferrooxidans. FEMS Microbiol. Lett. 97, 167-172. doi: 10.1111/j.1574-6968.1992.tb05457.x

Dopson, M. (2016). "Physiological and phylogenetic diversity of acidophilic bacteria," in Acidophiles: Life in Extremely Acidic Environments, eds R. Quatrini and D. B. Johnson (Poole: Caister Academic Press), 79-92.

Dopson, M., Baker-Austin, C., and Bond, P. (2007). Towards determining details of anaerobic growth coupled to ferric iron reduction by the acidophilic archaeon 'Ferroplasma acidarmanus' Fer1. Extremophiles 11, 159-168. doi: 10.1007/ s00792-006-0029-y

Dopson, M., Baker-Austin, C., Hind, A., Bowman, J. P., and Bond, P. L. (2004). Characterization of Ferroplasma isolates and Ferroplasma acidarmanus sp. nov., extreme acidophiles from acid mine drainage and industrial bioleaching environments. Appl. Environ. Microbiol. 70, 2079-2088. doi: 10.1128/AEM.70. 4.2079-2088.2004

du Plessis, C. A., Slabbert, W., Hallberg, K. B., and Johnson, D. B. (2011). Ferredox: a biohydrometallurgical processing concept for limonitic nickel laterites. Hydrometallurgy 109, 221-229. doi: 10.1016/j.hydromet.2011.07.005

Ehrenreich, A., and Widdel, F. (1994). Anaerobic oxidation of ferrous iron by purple bacteria, a new type of phototrophic metabolism. Appl. Environ. Microbiol. 60, 4517-4526. doi: 10.1128/aem.60.12.4517-4526.1994

Elbehti, A., Brasseur, G., and Lemesle-Meunier, D. (2000). First evidence for existence of an uphill electron transfer through the $b c_{1}$ and NADH-Q oxidoreductase complexes of the acidophilic obligate chemolithotrophic ferrous ion-oxidizing bacterium Thiobacillus ferrooxidans. J. Bacteriol. 182, 3602-3606. doi: 10.1128/JB.182.12.3602-3606.2000

Elbehti, A., Nitschke, W., Tron, P., Michel, C., and Lemesle-Meunier, D. (1999). Redox components of cytochrome $b c$-type enzymes in acidophilic prokaryotes. I. Characterization of the cytochrome $b c_{1}$-type complex of the acidophilic ferrous ion-oxidizing bacterium Thiobacillus ferrooxidans. J. Biol. Chem. 274, 16760-16765. doi: 10.1074/jbc.274.24.16760

Emerson, D., Fleming, E. J., and McBeth, J. M. (2010). Iron-oxidizing bacteria: an environmental and genomic perspective. Annu. Rev. Microbiol. 64, 561-583. doi: 10.1146/annurev.micro.112408.134208

Falagán, C., Foesel, B., and Johnson, B. (2017). Acidicapsa ferrireducens sp. nov., Acidicapsa acidiphila sp. nov., and Granulicella acidiphila sp. nov. novel acidobacteria isolated from metal-rich acidic waters. Extremophiles 21, 459-469. doi: 10.1007/s00792-017-0916-4

Falagán, C., and Johnson, D. B. (2014). Acidibacter ferrireducens gen. nov., sp. nov. an acidophilic ferric iron-reducing gammaproteobacterium. Extremophiles 18, 1067-1073. doi: 10.1007/s00792-014-0684-3

Falagán, C., and Johnson, D. B. (2016). Acidithiobacillus ferriphilus sp. nov., a facultatively anaerobic iron- and sulfur-metabolizing extreme acidophile. Int. J. Syst. Evol. Microbiol. 66, 206-211. doi: 10.1099/ijsem.0.000698

Ferrer, A., Orellana, O., and Levicán, G. (2016). "Oxidative stress and metal tolerance in extreme acidophiles," in Acidophiles: Life in Extremely Acidic Environments, eds R. Quatrini and D. B. Johnson (Norfolk: Caister Academic Press), 63-76. 
Gadd, G. M. (2009). "Heavy metal pollutants: environmental and biotechnological aspects," in Encyclopedia of Microbiology, ed. M. Schaechter (Oxford: Elsevier), 321-334.

Gentina, J. C., and Acevedo, F. (2016). Copper bioleaching in Chile. Minerals 6:23. doi: $10.3390 / \min 6010023$

Giaveno, M. A., Urbieta, M. S., Ulloa, J. R., Toril, E. G., and Donati, E. R. (2013). Physiologic versatility and growth flexibility as the main characteristics of a novel thermoacidophilic Acidianus strain isolated from Copahue geothermal area in Argentina. Microb. Ecol. 65, 336-346. doi: 10.1007/s00248-012-0129-4

Giudici-Orticoni, M. T., Guerlesquin, F., Bruschi, M., and Nitschke, W. (1999). Interaction-induced redox switch in the electron transfer complex rusticyanincytochrome $c_{4}$. J. Biol. Chem. 274, 30365-30369. doi: 10.1074/jbc.274.43. 30365

Golyshina, O. V., Ferrer, M., and Golyshin, P. N. (2016). "Diversity and physiologies of acidophilic Archaea," in Acidophiles: Life in Extremely Acidic Environments, eds R. Quatrini and D. B. Johnson (Norfolk: Caister Academic Press), 93-106.

Golyshina, O. V., Yakimov, M. M., Lünsdorf, H., Ferrer, M., Nimtz, M., Timmis, K. N., et al. (2009). Acidiplasma aeolicum gen. nov., sp. nov., a euryarchaeon of the family Ferroplasmaceae isolated from a hydrothermal pool, and transfer of Ferroplasma cupricumulans to Acidiplasma cupricumulans comb. nov. Int. J. Syst. Evol. Microbiol. 59, 2815-2823. doi: 10.1099/ijs.0.009639-0

González, D., Huber, K. J., Tindall, B., Hedrich, S., Rojas-Villalobos, C., Quatrini, R., et al. (2020). Acidiferrimicrobium australe gen. nov., sp. nov., an acidophilic and obligately heterotrophic, member of the Actinobacteria that catalyses dissimilatory oxido-reduction of iron isolated from metal-rich acidic water in Chile. Int. J. Syst. Evol. Microbiol. 70, 3348-3354. doi: 10.1099/ijsem.0.004179

González-Toril, E., Llobet-Brossa, E., Casamayor, E. O., Amann, R., and Amils, R. (2003). Microbial ecology of an extreme acidic environment, the Tinto River. Appl. Environ. Microbiol. 69, 4853-4865. doi: 10.1128/AEM.69.8.4853-4865. 2003

Gyure, R. A., Konopka, A., Brooks, A., and Doemel, W. (1990). Microbial sulfate reduction in acidic ( $\mathrm{pH} 3$ ) strip-mine lakes. FEMS Microbiol. Lett. 73, 193-201. doi: 10.1111/j.1574-6968.1990.tb03941.x

Hallberg, K. B., González-Toril, E., and Johnson, D. B. (2010). Acidithiobacillus ferrivorans, sp. nov.; facultatively anaerobic, psychrotolerant iron-, and sulfuroxidizing acidophiles isolated from metal mine-impacted environments. Extremophiles 14, 9-19. doi: 10.1007/s00792-009-0282-y

Hallberg, K. B., Grail, B. M., du Plessis, C. A., and Johnson, D. B. (2011a). Reductive dissolution of ferric iron minerals: a new approach for bio-processing nickel laterites. Miner. Eng. 24, 620-624. doi: 10.1016/j.mineng.2010.09.005

Hallberg, K. B., Hedrich, S., and Johnson, D. B. (2011b). Acidiferrobacter thiooxydans, gen. nov. sp. nov.; an acidophilic, thermo-tolerant, facultatively anaerobic iron- and sulfur-oxidizer of the family Ectothiorhodospiraceae. Extremophiles 15, 271-279. doi: 10.1007/s00792-011-0359-2

Hawkes, R. B., Franzmann, P. D., O’Hara, G., and Plumb, J. J. (2006). Ferroplasma cupricumulans sp. nov., a novel moderately thermophilic, acidophilic archaeon isolated from an industrial-scale chalcocite bioleach heap. Extremophiles 10, 525-530. doi: 10.1007/s00792-006-0527-y

Hedrich, S., and Johnson, D. B. (2013b). Aerobic and anaerobic oxidation of hydrogen by acidophilic bacteria. FEMS Microbiol. Lett. 349, 40-45. doi: 10. 1111/1574-6968.12290

Hedrich, S., and Johnson, D. B. (2013a). Acidithiobacillus ferridurans sp. nov., an acidophilic iron-, sulfur- and hydrogen-metabolizing chemolithotrophic gammaproteobacterium. Int. J. Syst. Evol. Microbiol. 63, 4018-4025. doi: 10. 1099/ijs.0.049759-0

Hedrich, S., Schlömann, M., and Johnson, D. B. (2011). The iron-oxidizing proteobacteria. Microbiology 157, 1551-1564. doi: 10.1099/mic.0.045344-0

Hofstetter, T. B., Heijman, C. G., Haderlein, S. B., Holliger, C., and Schwarzenbach, R. P. (1999). Complete reduction of TNT and other (poly)nitroaromatic compounds under iron-reducing subsurface conditions. Environ. Sci. Technol. 33, 1479-1487. doi: 10.1021/es9809760

Ilbert, M., and Bonnefoy, V. (2013). Insight into the evolution of the iron oxidation pathways. Biochim. Biophys. Acta 1827, 161-175. doi: 10.1016/j.bbabio.2012.10. 001

Ingledew, W. J. (1982). Thiobacillus ferrooxidans the bioenergetics of an acidophilic chemolithotroph. Biochim. Biophys. Acta 683, 89-117. doi: 10.1016/03044173(82)90007-6
Itoh, T., Yamanoi, K., Kudo, T., Ohkuma, M., and Takashina, T. (2011). Aciditerrimonas ferrireducens gen. nov., sp. nov., an iron-reducing thermoacidophilic actinobacterium isolated from a solfataric field. Int. J. Syst. Evol. Microbiol. 61, 1281-1285. doi: 10.1099/ijs.0.023044-0

Jiang, V., Khare, S. D., and Banta, S. (2021). Computational structure prediction provides a plausible mechanism for electron transfer by the outer membrane protein Cyc2 from Acidithiobacillus ferrooxidans. Protein Sci. 30, 1640-1652. doi: 10.1002/pro.4106

Johnson, D. B. (1998). Biodiversity and ecology of acidophilic microorganisms. FEMS Microbiol. Ecol. 27, 307-317. doi: 10.1111/j.1574-6941.1998.tb00547.x

Johnson, D. B. (2009). "Extremophiles: acidic environments," in Encyclopedia of Microbiology, ed. M. Schaechter (Oxford: Elsevier), 107-126.

Johnson, D. B. (2010). “The biogeochemistry of biomining," in Geomicrobiology: Molecular and Environmental Perspective, eds L. L. Barton, M. Mandl, and A. Loy (Dordrecht: Springer), 401-426.

Johnson, D. B. (2014). Biomining - biotechnologies for extracting and recovering metals from ores and waste materials. Curr. Opin. Biotechnol. 30, 24-31. doi: 10.1016/j.copbio.2014.04.008

Johnson, D. B., Bacelar-Nicolau, P., Okibe, N., Thomas, A., and Hallberg, K. B. (2009). Ferrimicrobium acidiphilum gen. nov., sp. nov. and Ferrithrix thermotolerans gen. nov., sp. nov. heterotrophic, iron-oxidizing, extremely acidophilic actinobacteria. Int. J. Syst. Evol. Microbiol. 59, 1082-1089. doi: 10. 1099/ijs.0.65409-0

Johnson, D. B., and Bridge, T. A. M. (2002). Reduction of ferric iron by acidophilic heterotrophic bacteria: evidence for constitutive and inducible enzyme systems in Acidiphilium spp. J. Appl. Microbiol. 92, 315-321. doi: 10.1046/j.1365-2672. 2002.01535.x

Johnson, D. B., and du Plessis, C. A. (2015). Biomining in reverse gear: using bacteria to extract metals from oxidised ores. Miner. Eng. 75, 2-5. doi: 10.1016/ j.mineng.2014.09.024

Johnson, D. B., Ghauri, M. A., and McGinness, S. (1993). Biogeochemical cycling of iron and sulphur in leaching environments. FEMS Microbiol. Rev. 11, 63-70. doi: 10.1111/j.1574-6976.1993.tb00268.x

Johnson, D. B., Grail, B. M., and Hallberg, K. B. (2013). A new direction for biomining: extraction of metals by reductive dissolution of oxidized ores. Minerals 3, 49-58. doi: 10.3390/min3010049

Johnson, D. B., and Hallberg, K. B. (2008). Carbon, iron and sulfur metabolism in acidophilic micro-organisms. Adv. Microb. Physiol. 54, 201-255.

Johnson, D. B., Hedrich, S., and Pakostova, E. (2017). Indirect redox transformations of iron, copper, and chromium catalyzed by extremely acidophilic bacteria. Front. Microbiol. 8:211. doi: 10.3389/fmicb.2017.0 0211

Johnson, D. B., Joulian, C., d'Hugues, P., and Hallberg, K. B. (2008). Sulfobacillus benefaciens sp. nov., an acidophilic facultative anaerobic Firmicute isolated from mineral bioleaching operations. Extremophiles 12, 789-798. doi: 10.1007/ s00792-008-0184-4

Johnson, D. B., Kanao, T., and Hedrich, S. (2012). Redox transformations of iron at extremely low pH: fundamental and applied aspects. Front. Microbiol. 3:96. doi: $10.3389 /$ fmicb. 2012.00096

Johnson, D. B., and McGinness, S. (1991). Ferric iron reduction by acidophilic heterotrophic bacteria. Appl. Environ. Microbiol. 57, 207-211. doi: 10.1128/aem. 57.1.207-211.1991

Johnson, D. B., and Pakostova, E. (2021). Dissolution of manganese (IV) oxide mediated by acidophilic bacteria, and demonstration that manganese (IV) can act as both a direct and indirect electron acceptor for iron-reducing Acidithiobacillus spp. Geomicrobiol. J. 38, 570-576. doi: 10.1080/01490451.2021. 1903624

Johnson, D. B., and Quatrini, R. (2020). Acidophile microbiology in space and time. Curr. Issues Mol. Biol. 39, 63-76. doi: 10.21775/cimb.039.063

Johnson, D. B., Smith, S. L., and Santos, A. L. (2021). Bioleaching of transition metals from limonitic laterite deposits and reassessment of the multiple roles of sulfur-oxidizing acidophiles in the process. Front. Microbiol. 12:703177. doi: 10.3389/fmicb.2021.703177

Johnson, D. B., Stallwood, B., Kimura, S., and Hallberg, K. B. (2006). Isolation and characterization of Acidicaldus organivorus, gen. nov., sp. nov. a novel sulfur-oxidizing, ferric iron-reducing thermo-acidophilic heterotrophic Proteobacterium. Arch. Microbiol. 185, 212-221. doi: 10.1007/s00203-0060087-7 
Jones, R. M., Hedrich, S., and Johnson, D. B. (2013). Acidocella aromatica sp. nov. an acidophilic heterotrophic Alphaproteobacterium with unusual phenotypic traits. Extremophiles 17, 841-850. doi: 10.1007/s00792-013-0566-0

Jones, R. M., and Johnson, D. B. (2015). Acidithrix ferrooxidans gen. nov., sp. nov.; a filamentous and obligately heterotrophic, acidophilic member of the Actinobacteria that catalyzes dissimilatory oxido-reduction of iron. Res. Microbiol. 166, 111-120. doi: 10.1016/j.resmic.2015.01.003

Kai, M., Yano, T., Tamegai, H., Fukumori, Y., and Yamanaka, T. (1992). Thiobacillus ferrooxidans cytochrome $c$ oxidase: purification, and molecular and enzymatic features. J. Biochem. 112, 816-821. doi: 10.1093/oxfordjournals. jbchem.a123982

Kappler, A., and Straub, K. L. (2005). Geomicrobiological cycling of iron. Rev. Mineral. Geochem. 59, 85-108. doi: 10.2138/rmg.2005.59.5

Karavaiko, G. I., Bogdanova, T. I., Tourova, T. P., Kondrat'eva, T. F., Tsaplina, I. A., Egorova, M. A., et al. (2005). Reclassification of 'Sulfobacillus thermosulfidooxidans subsp. thermotolerans' strain $\mathrm{K} 1$ as Alicyclobacillus tolerans sp. nov. and Sulfobacillus disulfidooxidans Dufresne et al. 1996 as Alicyclobacillus disulfidooxidans comb. nov., and emended description of the genus Alicyclobacillus. Int. J. Syst. Evol. Microbiol. 55, 941-947. doi: 10.1099/ijs. $0.63300-0$

Kashefi, K., Holmes, D. E., Lovley, D. R., and Tor, J. M. (2004). "Potential importance of dissimilatory $\mathrm{Fe}(\mathrm{III})$-reducing microorganisms in hot sedimentary environments," in The Subseafloor Biosphere at Mid-Ocean Ridges, eds W. S. Wilcock, E. F. DeLong, D. S. Kelley, J. A. Baross, and S. Craig Cary (Washington, DC: American Geophysical Union), 199-211.

Kucera, J., Bouchal, P., Cerna, H., Potesil, D., Janiczek, O., Zdrahal, Z., et al. (2012). Kinetics of anaerobic elemental sulfur oxidation by ferric iron in Acidithiobacillus ferrooxidans and protein identification by comparative 2-DEMS/MS. Antonie Van Leeuwenhoek 101, 561-573. doi: 10.1007/s10482-0119670-2

Kucera, J., Bouchal, P., Lochman, J., Potesil, D., Janiczek, O., Zdrahal, Z., et al. (2013). Ferrous iron oxidation by sulfur-oxidizing Acidithiobacillus ferrooxidans and analysis of the process at the levels of transcription and protein synthesis. Antonie Van Leeuwenhoek 103, 905-919. doi: 10.1007/s10482-0129872-2

Kucera, J., Lochman, J., Bouchal, P., Pakostova, E., Mikulasek, K., Hedrich, S., et al. (2020). A model of aerobic and anaerobic metabolism of hydrogen in the extremophile Acidithiobacillus ferrooxidans. Front. Microbiol. 11:610836. doi: 10.3389/fmicb.2020.610836

Kucera, J., Pakostova, E., Janiczek, O., and Mandl, M. (2015). Changes in Acidithiobacillus ferrooxidans ability to reduce ferric iron by elemental sulfur. Adv. Mat. Res. 1130, 97-100.

Kucera, J., Pakostova, E., Lochman, J., Janiczek, O., and Mandl, M. (2016a). Are there multiple mechanisms of anaerobic sulfur oxidation with ferric iron in Acidithiobacillus ferrooxidans? Res. Microbiol. 167, 357-366. doi: 10.1016/j. resmic.2016.02.004

Kucera, J., Sedo, O., Potesil, D., Janiczek, O., Zdrahal, Z., and Mandl, M. (2016b). Comparative proteomic analysis of sulfur-oxidizing Acidithiobacillus ferrooxidans CCM 4253 cultures having lost the ability to couple anaerobic elemental sulfur oxidation with ferric iron reduction. Res. Microbiol. 167, 587-594. doi: 10.1016/j.resmic.2016.06.009

Küsel, K., Roth, U., and Drake, H. L. (2002). Microbial reduction of Fe(III) in the presence of oxygen under low pH conditions. Environ. Microbiol. 4, 414-421. doi: 10.1046/j.1462-2920.2002.00314.x

Lovley, D. R. (1991). Dissimilatory Fe(III) and Mn(IV) reduction. Microbiol. Rev. 55, 259-287. doi: 10.1128/mr.55.2.259-287.1991

Lu, S., Gischkat, S., Reiche, M., Akob, D. M., Hallberg, K. B., and Küsel, K. (2010). Ecophysiology of Fe-cycling bacteria in acidic sediments. Appl. Environ. Microbiol. 76, 8174-8183. doi: 10.1128/AEM.01931-10

Malarte, G., Leroy, G., Lojou, E., Abergel, C., Bruschi, M., and Giudici-Orticoni, M. T. (2005). Insight into molecular stability and physiological properties of the diheme cytochrome $\mathrm{CYC}_{41}$ from the acidophilic bacterium Acidithiobacillus ferrooxidans. Biochemistry 44, 6471-6481. doi: 10.1021/bi048425b

Matin, A. (1999). "pH homeostasis in acidophiles," in Novartis Foundation Symposium 221 - Bacterial Responses to $\mathrm{pH}$, eds D. J. Chadwick and G. Cardew (New York, NY: Wiley). doi: 10.1002/9780470515631.ch10

Marrero, J., Coto, O., Goldmann, S., Graupner, T., and Schippers, A. (2015). Recovery of nickel and cobalt from laterite tailings by reductive dissolution under aerobic conditions using Acidithiobacillus species. Environ. Sci. Technol. 49, 6674-6682. doi: 10.1021/acs.est.5b00944

Marrero, J., Coto, O., and Schippers, A. (2017). Anaerobic and aerobic reductive dissolutions of iron-rich nickel laterite overburden by Acidithiobacillus. Hydrometallurgy 168, 49-55. doi: 10.1016/j.hydromet.2016.08.012

Masaki, Y., Tsutsumi, K., and Okibe, N. (2018). Iron redox transformation by the thermo-acidophilic archaea from the genus Sulfolobus. Geomicrobiol. J. 35, 757-767. doi: 10.1080/01490451.2018.1465491

Nancucheo, I., Grail, B. M., Hilario, F., du Plessis, C. A., and Johnson, D. B. (2014). Extraction of copper from an oxidized (lateritic) ore using bacterially catalysed reductive dissolution. Appl. Microbiol. Biotechnol. 98, 6297-6305. doi: 10.1007/s00253-014-5687-6

Nancucheo, I., Oliveira, G., Lopes, M., and Johnson, D. B. (2019). Bioreductive dissolution as a pretreatment for recalcitrant rare-earth phosphate minerals associated with lateritic ores. Minerals 9:136. doi: 10.3390/min9030136

Norris, P. R., Falagán, C., Moya-Beltrán, A., Castro, M., Quatrini, R., and Johnson, D. B. (2020). Acidithiobacillus ferrianus sp. nov. an ancestral extremely acidophilic and facultatively anaerobic chemolithoautotroph. Extremophiles 24, 329-337. doi: 10.1007/s00792-020-01157-1

Norris, P. R., Gould, O. J. P., and Ogden, T. J. (2015). Iron solubilization during anaerobic growth of acidophilic microorganisms with a polymetallic sulfide ore. Miner. Eng. 75, 77-84. doi: 10.1016/j.mineng.2014.12.004

Norris, P. R., Laigle, L., and Slade, S. (2018). Cytochromes in anaerobic growth of Acidithiobacillus ferrooxidans. Microbiology 164, 383-394. doi: 10.1099/mic.0. 000616

Ohmura, N., Sasaki, K., Matsumoto, N., and Saiki, H. (2002). Anaerobic respiration using $\mathrm{Fe}^{3+}, \mathrm{S}^{0}$, and $\mathrm{H}_{2}$ in the chemolithoautotrophic bacterium Acidithiobacillus ferrooxidans. J. Bacteriol. 184, 2081-2087. doi: 10.1128/JB.184. 8.2081-2087.2002

Osorio, H., Mangold, S., Denis, Y., Nancucheo, I., Esparza, M., Johnson, D. B., et al. (2013). Anaerobic sulfur metabolism coupled to dissimilatory iron reduction in the extremophile Acidithiobacillus ferrooxidans. Appl. Environ. Microbiol. 79, 2172-2181. doi: 10.1128/AEM.03057-12

Ottow, J. C. G., and Glathe, H. (1971). Isolation and identification of ironreducing bacteria from gley soils. Soil Biol. Biochem. 3, 43-55. doi: 10.1016/ 0038-0717(71)90030-7

Plumb, J. J., Haddad, C. M., Gibson, J. A. E., and Franzmann, P. D. (2007). Acidianus sulfidivorans sp. nov., an extremely acidophilic, thermophilic archaeon isolated from a solfatara on Lihir Island, Papua New Guinea, and emendation of the genus description. Int. J. Syst. Evol. Microbiol. 57, 1418-1423. doi: 10.1099/ijs.0.64846-0

Pronk, J. T., Bruyn, J. C., de Bos, P., and Kuenen, J. G. (1992). Anaerobic growth of Thiobacillus ferrooxidans. Appl. Environ. Microbiol. 58:2227. doi: 10.1128/aem. 58.7.2227-2230.1992

Pronk, J. T., Liem, K., Bos, P., and Kuenen, J. G. (1991). Energy transduction by anaerobic ferric iron respiration in Thiobacillus ferrooxidans. Appl. Environ. Microbiol. 57:2063. doi: 10.1128/aem.57.7.2063-2068.1991

Quatrini, R., Appia-Ayme, C., Denis, Y., Ratouchniak, J., Veloso, F., Valdes, J., et al. (2006). Insights into the iron and sulfur energetic metabolism of Acidithiobacillus ferrooxidans by microarray transcriptome profiling. Hydrometallurgy 83, 263-272. doi: 10.1016/j.hydromet.2006.03.030

Quatrini, R., and Johnson, D. B. (2016). Acidophiles: Life in Extremely Acidic Environments. Norfolk: Caister Academic Press.

Rohwerder, T. (2002). Untersuchungen zur Enzymatischen Oxidation von Elementarschwefel bei Acidophilen Laugungsbakterien. Thesis. Hamburg: Universität Hamburg.

Sakai, H. D., and Kurosawa, N. (2018). Saccharolobus caldissimus gen. nov., sp. nov., a facultatively anaerobic iron-reducing hyperthermophilic archaeon isolated from an acidic terrestrial hot spring, and reclassification of Sulfolobus solfataricus as Saccharolobus solfataricus comb. nov. and Sulfolobus shibatae as Saccharolobus shibatae comb. nov. Int. J. Syst. Evol. Microbiol. 68, 1271-1278. doi: 10.1099/ijsem.0.002665

Sand, W. (1989). Ferric iron reduction by Thiobacillus ferrooxidans at extremely low pH-values. Biogeochemistry 7, 195-201. doi: 10.1007/BF00004217

Santos, A. L., Dybowska, A., Schofield, P. F., Herrington, R. J., and Johnson, D. B. (2020). Sulfur-enhanced reductive bioprocessing of cobalt-bearing materials for base metals recovery. Hydrometallurgy 195:105396. doi: 10.1016/j.hydromet. 2020.105396 
Slobodkin, A. I. (2005). Thermophilic microbial metal reduction. Microbiology 74, 501-514. doi: 10.1007/s11021-005-0096-6

Smith, S. L., Grail, B. M., and Johnson, D. B. (2017). Reductive bioprocessing of cobalt-bearing limonitic laterites. Miner. Eng. 106, 86-90. doi: 10.1016/j. mineng.2016.09.009

Smith, S. L., and Johnson, D. B. (2018). Growth of Leptospirillum ferriphilum in sulfur medium in co-culture with Acidithiobacillus caldus. Extremophiles 22, 327-333. doi: 10.1007/s00792-018-1001-3

Stolz, A. (2017). "Acidophile," in Extremophile Mikroorganismen, ed. A. Stolz (Berlin: Springer), 79-106.

Sugio, T., Domatsu, C., Munakata, O., Tano, T., and Imai, K. (1985). Role of a ferric ion-reducing system in sulfur oxidation of Thiobacillus ferrooxidans. Appl. Environ. Microbiol. 49, 1401-1406. doi: 10.1128/aem.49.6.1401-1406.1985

Sugio, T., Mizunashi, W., Inagaki, K., and Tano, T. (1987). Purification and some properties of sulfur:ferric ion oxidoreductase from Thiobacillus ferrooxidans. J. Bacteriol. 169, 4916-4922. doi: 10.1128/jb.169.11.4916-4922.1987

Sugio, T., Taha, T. M., and Takeuchi, F. (2009). Ferrous iron production mediated by tetrathionate hydrolase in tetrathionate-, sulfur-, and iron-grown Acidithiobacillus ferrooxidans ATCC 23270 cells. Biosci. Biotechnol. Biochem. 73, 1381-1386. doi: 10.1271/bbb.90036

Sugio, T., Wada, K., Mori, M., Inagaki, K., and Tano, T. (1988). Synthesis of an iron-oxidizing system during growth of Thiobacillus ferrooxidans on sulfurbasal salts medium. Appl. Environ. Microbiol. 54, 150-152. doi: 10.1128/aem. 54.1.150-152.1988

Tsuboi, K., Sakai, H. D., Nur, N., Stedman, K. M., Kurosawa, N., and Suwanto, A. (2018). Sulfurisphaera javensis sp. nov., a hyperthermophilic and acidophilic archaeon isolated from Indonesian hot spring, and reclassification of Sulfolobus tokodaii Suzuki et al. 2002 as Sulfurisphaera tokodaii comb. nov. Int. J. Syst. Evol. Microbiol. 68, 1907-1913. doi: 10.1099/ijsem.0.002765

Walker, J. C. G. (1987). Was the Archaean biosphere upside down? Nature 329, 710-712. doi: 10.1038/329710a0

Wang, R., Lin, J.-Q., Liu, X.-M., Pang, X., Zhang, C.-J., Yang, C.-L., et al. (2019). Sulfur oxidation in the acidophilic autotrophic Acidithiobacillus spp. Front. Microbiol. 9:3290. doi: 10.3389/fmicb.2018.03290

Yarzábal, A., Brasseur, G., Ratouchniak, J., Lund, K., Lemesle-Meunier, D., DeMoss, J. A., et al. (2002). The high-molecular-weight cytochrome $c$ Cyc2 of
Acidithiobacillus ferrooxidans is an outer membrane protein. J. Bacteriol. 184, 313-317. doi: 10.1128/JB.184.1.313-317.2002

Yoshida, N., Nakasato, M., Ohmura, N., Ando, A., Saiki, H., Ishii, M., et al. (2006). Acidianus manzaensis sp. nov., a novel thermoacidophilic archaeon growing autotrophically by the oxidation of $\mathrm{H}_{2}$ with the reduction of $\mathrm{Fe}^{3+}$. Curr. Microbiol. 53, 406-411. doi: 10.1007/s00284-006-0151-1

Zhang, R., Hedrich, S., Jin, D., Breuker, A., and Schippers, A. (2021). Sulfobacillus harzensis sp. nov., an acidophilic bacterium inhabiting mine tailings from a polymetallic mine. Int. J. Syst. Evol. Microbiol. 71:4871. doi: 10.1099/ijsem.0. 004871

Zhang, R., Hedrich, S., Ostertag-Henning, C., and Schippers, A. (2018). Effect of elevated pressure on ferric iron reduction coupled to sulfur oxidation by biomining microorganisms. Hydrometallurgy 178, 215-223. doi: 10.1016/j. hydromet.2018.05.003

Zhou, H., Zhang, R., Hu, P., Zeng, W., Xie, Y., Wu, C., et al. (2008). Isolation and characterization of Ferroplasma thermophilum sp. nov., a novel extremely acidophilic, moderately thermophilic archaeon and its role in bioleaching of chalcopyrite. J. Appl. Microbiol. 105, 591-601. doi: 10.1111/j.1365-2672.2008. 03807.x

Conflict of Interest: The authors declare that the research was conducted in the absence of any commercial or financial relationships that could be construed as a potential conflict of interest.

Publisher's Note: All claims expressed in this article are solely those of the authors and do not necessarily represent those of their affiliated organizations, or those of the publisher, the editors and the reviewers. Any product that may be evaluated in this article, or claim that may be made by its manufacturer, is not guaranteed or endorsed by the publisher.

Copyright (C) 2022 Malik and Hedrich. This is an open-access article distributed under the terms of the Creative Commons Attribution License (CC BY). The use, distribution or reproduction in other forums is permitted, provided the original author(s) and the copyright owner(s) are credited and that the original publication in this journal is cited, in accordance with accepted academic practice. No use, distribution or reproduction is permitted which does not comply with these terms. 